\title{
Structural basis of tubulin recruitment and assembly by microtubule polymerases with tumor overexpressed gene (TOG) domain arrays
}

\author{
Stanley Nithianantham ${ }^{1}$, Brian D Cook', Madeleine Beans', Fei Guo1, \\ Fred Chang ${ }^{2}$, Jawdat Al-Bassam ${ }^{1 *}$
}

${ }^{1}$ Molecular Cellular Biology Department, University of California, Davis, United States; ${ }^{2}$ Department of Cell and Tissue Biology, University of California, San Francisco, United States

*For correspondence: jawdat@ucdavis.edu

Competing interests: The authors declare that no competing interests exist.

Funding: See page 30

Received: 05 June 2018

Accepted: 31 October 2018

Published: 13 November 2018

Reviewing editor: Thomas Surrey, The Francis Crick Institute, United Kingdom

(c) Copyright Nithianantham et al. This article is distributed under the terms of the Creative Commons Attribution License, which permits unrestricted use and redistribution provided that the original author and source are credited.

\begin{abstract}
XMAP215/Stu2/Alp14 proteins accelerate microtubule plus-end polymerization by recruiting tubulins via arrays of tumor overexpressed gene (TOG) domains, yet their mechanism remains unknown. Here, we describe the biochemical and structural basis for TOG arrays in recruiting and polymerizing tubulins. Alp14 binds four tubulins via dimeric TOG1-TOG2 subunits, in which each domain exhibits a distinct exchange rate for tubulin. X-ray structures revealed squareshaped assemblies composed of pseudo-dimeric TOG1-TOG2 subunits assembled head-to-tail, positioning four unpolymerized tubulins in a polarized wheel-like configuration. Crosslinking and electron microscopy show Alp14-tubulin forms square assemblies in solution, and inactivating their interfaces destabilize this organization without influencing tubulin binding. An X-ray structure determined using approach to modulate tubulin polymerization revealed an unfurled assembly, in which TOG1-TOG2 uniquely bind to two polymerized tubulins. Our findings suggest a new microtubule polymerase model in which TOG arrays recruit tubulins by forming square assemblies that then unfurl, facilitating their concerted polymerization into protofilaments.
\end{abstract}

DOI: https://doi.org/10.7554/eLife.38922.001

\section{Introduction}

Microtubules (MTs) are highly dynamic polarized polymers that perform critical and diverse cellular functions including formation of bipolar mitotic spindles, intracellular organization, and modulation of cell development and cell migration (Akhmanova and Steinmetz, 2008; Akhmanova and Steinmetz, 2015). MTs are assembled from $\alpha \beta$-tubulin heterodimers (herein termed $\alpha \beta$-tubulin), and their polymerization exhibits dynamic instability arising from guanosine triphosphate (GTP) hydrolysis in $\beta$ tubulins at MT ends. However, the conformational changes promoting soluble $\alpha \beta$-tubulins to polymerize at MT ends remain poorly understood. Polymerization of $\alpha \beta$-tubulin and GTP hydrolysis are regulated by conserved proteins that bind at MT plus-ends or along MT lattices (Akhmanova and Steinmetz, 2008; Akhmanova and Steinmetz, 2011; Akhmanova and Steinmetz, 2015; AlBassam and Chang, 2011; Al-Bassam et al., 2010; Brouhard and Rice, 2014). The XMAP215/Stu2/ Alp14 MT polymerases are among the best-studied families of MT regulators. They localize to the extreme tips of MT plus-ends and accelerate $\alpha \beta$-tubulin polymerization in eukaryotes (Akhmanova and Steinmetz, 2011; Akhmanova and Steinmetz, 2015; Al-Bassam and Chang, 2011; Maurer et al., 2014). Loss or depletion of MT polymerases is lethal in most eukaryotes as it severely decreases MT polymerization rates during interphase, resulting in shortened mitotic spindles (Al-Bassam et al., 2012; Cullen et al., 1999; Wang and Huffaker, 1997). MT polymerases also 
bind kinetochores, where they accelerate MT dynamics and regulate kinetochore-MT attachment (Miller et al., 2016; Tanaka et al., 2005). MT polymerases recruit $\alpha \beta$-tubulins via arrays of conserved tumor overexpressed gene (TOG) domains (herein termed TOG arrays), which are critical for their function (Reber et al., 2013; Widlund et al., 2011). Arrays of TOG-like domains are conserved in two other classes of MT regulators, CLASP and Crescerin/CHE-12 protein families (Al-Bassam and Chang, 2011; Al-Bassam et al., 2010; Das et al., 2015), suggesting that arrays of TOG domains uniquely evolved to regulate diverse MT polymerization functions through the binding of $\alpha \beta$-tubulins in various intracellular settings.

Yeast MT polymerases, such as Saccharomyces cerevisiae Stu2p and Schizosccharomyces pombe Alp14, are homodimers containing two unique and conserved TOG domain classes, TOG1 and TOG2, per subunit, numbered based on their proximity to the $\mathrm{N}$-terminus in the protein sequence. In contrast, metazoan orthologs, such as XMAP215 and ch-TOG, are monomers with five tandem TOG domains, TOG1 through TOG5 (Al-Bassam and Chang, 2011; Brouhard and Rice, 2014). Phylogenetic analyses suggest that TOG1 and TOG2 domains are evolutionarily distinct (Al-Bassam and Chang, 2011), and that TOG3 and TOG4 domains in metazoans are evolutionarily and structurally exclusively related to the TOG1 and TOG2 domains, respectively (Brouhard et al., 2008; Fox et al., 2014; Howard et al., 2015). Thus, despite differences in TOG array organization in yeast and metazoan proteins, both groups contain an array of tandem TOG1-TOG2 domains.

Structural studies contributed to our understanding of the molecular basis of TOG domain function in recruiting soluble $\alpha \beta$-tubulin. Each TOG domain is composed of six $\alpha$-helical HEAT (Huntingtin, EF3A, ATM, and TOR) repeats, which form a conserved paddle-shaped structure (AlBassam and Chang, 2011; Al-Bassam et al., 2007; Al-Bassam et al., 2006; Brouhard and Rice, 2014; Slep and Vale, 2007). X-ray structures of isolated TOG1 and TOG2 domains in complex with $\alpha \beta$-tubulins revealed that these domains recognize the curved $\alpha \beta$-tubulin conformations via interhelical loops positioned along an edge of these paddle-shaped domains (Ayaz et alo, 2014; Ayaz et al., 2012). Straightening of the curved $\alpha \beta$-tubulins upon polymerization into MTs likely dissociates TOG domains from the complexes. Our previous studies indicate that native TOG arrays from yeast or metazoan MT polymerases assemble into discrete particle-like assemblies upon binding $\alpha \beta$-tubulin (Al-Bassam and Chang, 2011; Al-Bassam et al., 2006). Both TOG1 and TOG2 domains are critical for MT polymerase function, and their inactivation results in MT functional defects (Al-Bassam et al., 2012; Al-Bassam et al., 2006; Ayaz et al., 2014). Two models were proposed to explain how arrays of TOG domains function as MT polymerases. One model based on studies of native TOG arrays indicates that they may form ordered assemblies upon binding $\alpha \beta$ tubulins (Al-Bassam et al., 2006; Brouhard et al., 2008). A second model, based on studies of isolated TOG domains or short TOG arrays, suggests that these arrays form flexible assemblies in which TOG1 and TOG2 independently recruit multiple $\alpha \beta$-tubulins to MT plus-ends (Al-Bassam and Chang, 2011; Ayaz et al., 2014). Distinguishing between these models requires understanding the high-resolution organization of native TOG arrays in complex with $\alpha \beta$-tubulin and their transitions during $\alpha \beta$-tubulin recruitment and polymerization phases.

Here, we describe biochemical and structural states of TOG arrays during $\alpha \beta$-tubulin recruitment and polymerization states. We show that the yeast MT polymerase, Alp14, recruits four $\alpha \beta$-tubulins using dimeric arrays of TOG1-TOG2 domains. TOG1 and TOG2 domains each bind and release $\alpha \beta$ tubulins with different rates. X-ray structures reveal pseudo-dimeric TOG1-TOG2 subunits in headto-tail square-shaped assemblies, each of which orients four unpolymerized $\alpha \beta$-tubulins in a polarized configuration. Crosslinking and mass spectrometry and electron microscopy studies show that dimeric yeast TOG arrays form these square assemblies in solution. Alp14 mutants with inactivated binding interfaces show disorganized configurations or polymerized arrangements, but without any defects in $\alpha \beta$-tubulin binding. Using a novel approach to promote the limited polymerization of $\alpha \beta$ tubulins while bound to TOG arrays, we determined an X-ray structure of an 'unfurled' TOG1-TOG2 array: $\alpha \beta$-tubulin assembly revealing TOG1 and TOG2 bound to two $\alpha \beta$-tubulins polymerized headto-tail into a protofilament. Our studies establish a new 'polarized unfurling' model for TOG arrays as MT polymerases. 


\section{Results}

\section{TOG1 and TOG2 domains possess distinct affinities for $\alpha \beta$-tubulin}

The S. pombe Alp14 is a typical yeast MT polymerase, consisting of a tandem array of N-terminal TOG1 and TOG2 domains separated by a 60-residue linker, followed by a Ser-Lys-rich (SK-rich) region and a $\mathrm{C}$-terminal coiled-coil domain that regulates dimerization (Figure 1A). We studied the $\alpha \beta$-tubulin binding capacities and stoichiometries of near native monomeric and dimeric Alp14, which both consist of TOG1-TOG2 arrays and differ by the presence of a C-terminal SK-rich region and dimerization coiled-coil domain. Using quantitative size-exclusion chromatography (SEC) with multi-angle light scattering (SEC-MALS), we measured the $\alpha \beta$-tubulin binding stoichiometry for monomeric Alp14 (herein termed Alp14-monomer: residues 1-510), and dimeric Alp14 (herein termed wt-Alp14-dimer: residues 1-690) at 80-100 mM KCl ionic strength (Figure 1A-B, Figure 1D; details described in Figure 1-figure supplement 1A-C,D-F; SEC-MALS control experiments shown in Figure 1-figure supplement 2G-H; Table 1) (Al-Bassam et al., 2012). We show that 1 $\mu \mathrm{M}$ wt-Alp14-dimer binds four $\alpha \beta$-tubulins per dimer via its four TOG domains (dimeric TOG1-TOG2 arrays), whereas $1 \mu \mathrm{M}$ wt-Alp14-monomer binds two $\alpha \beta$-tubulins per dimer via two TOG domains. Thus, TOG1 and TOG2 independently recruit $\alpha \beta$-tubulins (Figure 1A-B,D; Figure 1-figure supplement 1A-C,D-F; Tables 1 and 2).

Since TOG1 and TOG2 domains bind $\alpha \beta$-tubulins via narrow and mostly ionic binding interfaces, we studied the effect of a moderate increase in ionic strength (100-200 mM KCl) on $\alpha \beta$-tubulin binding capacities of TOG1 and TOG2 domains in these arrays (Ayaz et al., 2014; Ayaz et al., 2012). At $200 \mathrm{mM} \mathrm{KCl}$, both $1 \mu \mathrm{M}$ wt-Alp14-monomer and wt-Alp14-dimer bound roughly half as much $\alpha \beta$ tubulin than at $80-100 \mathrm{mM} \mathrm{KCl}$. Thus, either TOG1 or TOG2 domains may lose part or all of their $\alpha \beta$-tubulin-binding capacity at $200 \mathrm{mM} \mathrm{KCl}$ (Figure 1B,D; Figure 1-figure supplement 1A-C,D-F and Tables 1 and 2). These differences between the $\alpha \beta$-tubulin-binding stoichiometries at 100 versus $200 \mathrm{mM} \mathrm{KCl}$ resolve discrepancies regarding Alp14- or Stu2- $\alpha \beta$-tubulin-binding stoichiometries reported previously (Ayaz et al., 2014; Al-Bassam et al., 2012; Al-Bassam et al., 2006).

We next determined if the $\alpha \beta$-tubulin-binding capacity of TOG 1 or TOG 2 changed within arrays due to a change in ionic strength from 100 to $200 \mathrm{mM} \mathrm{KCl}$. Using SEC and SEC-MALs, we studied the $\alpha \beta$-tubulin-binding stoichiometry for Alp14-dimer mutants in which either TOG1 (termed TOG1M) or TOG2 (termed TOG2M) were inactivated through multiple-residue mutations in the $\alpha \beta$ tubulin-binding interfaces (Al-Bassam et al., 2007; Ayaz et al., 2012); see Materials and methods; Figure 1C, Figure 1D; Figure 1-figure supplement 1G-L). $1 \mu \mathrm{M}$ TOG1M, which only includes two active TOG2 domains, bound two $\alpha \beta$-tubulins at $100 \mathrm{mM} \mathrm{KCl}$, but almost completely dissociated from $\alpha \beta$-tubulin at $200 \mathrm{mM} \mathrm{KCl}$. TOG1M and $\alpha \beta$-tubulin did not co-migrate on SEC, and most of the $\alpha \beta$-tubulin migrated as a separate peak at $200 \mathrm{mM} \mathrm{KCl}$ (Figure 1D, Figure 1-figure supplement 1G-I; Tables 1 and 2). In contrast, TOG2M, which only includes two active TOG1 domains, bound and co-migrated with $\alpha \beta$-tubulin in both 100 and $200 \mathrm{mM} \mathrm{KCl}$ conditions (Figure 1D, Figure 1-figure supplement $1 \mathrm{~J}-L$, Tables 1 and 2). Moreover, molar ratios of $\alpha \beta$-tubulin bound to TOG1M and TOG2M measured by quantitative-SEC at $100 \mathrm{mM} \mathrm{KCl}$ and maximal $\alpha \beta$-tubulin stoichiometry determined by SEC-MALS at $80 \mathrm{mM} \mathrm{KCl}$ were roughly half of that measured for wt-Alp14-dimer at 80$100 \mathrm{mM} \mathrm{KCl}$ (Figure 1C,D). These data support the independent and distinct affinities of TOG1 and TOG2 domains in recruiting $\alpha \beta$-tubulins while within either monomeric or dimeric arrays, and that dimerization does not change the $\alpha \beta$-tubulin-binding stoichiometry. TOG1 and TOG2 showed distinct $\alpha \beta$-tubulin binding behaviors at 100 and $200 \mathrm{mM} \mathrm{KCl}$ (Figure 1F; the SEC-MALS controls are shown in Figure 1-figure supplement 2G)

Next, we quantitatively measured the absolute TOG1 and TOG2 binding affinities for $\alpha \beta$-tubulin using isothermal titration calorimetery (ITC), and determined how these affinities were influenced by changes in ionic strength at 100-200 mM KCl. ITC data showed that isolated TOG1 (residues 1-270) and TOG2 (residues 320-510) bound $\alpha \beta$-tubulins with roughly 2.5 -fold difference in dissociation constants (Figure 1E; Figure 1-figure supplement 3). At $100 \mathrm{mM} \mathrm{KCl}$, the dissociation constants for TOG1 and TOG2 were measured at 70 and $173 \mathrm{nM}$, respectively. These data suggest that both TOG1 and TOG2 exhibit a moderately high affinity for $\alpha \beta$-tubulin with a 2.5-fold difference in affinity, nearly identical to that previously reported (Ayaz et al., 2014; Ayaz et al., 2012). However, at $200 \mathrm{mM} \mathrm{KCl}$, we measured TOG1 and TOG2 $\alpha \beta$-tubulin dissociation constants at $1.5 \mu \mathrm{M}$ and 3.2 $\mu \mathrm{M}$, respectively, which showed 20 -fold weaker affinity in absolute values compared to those 


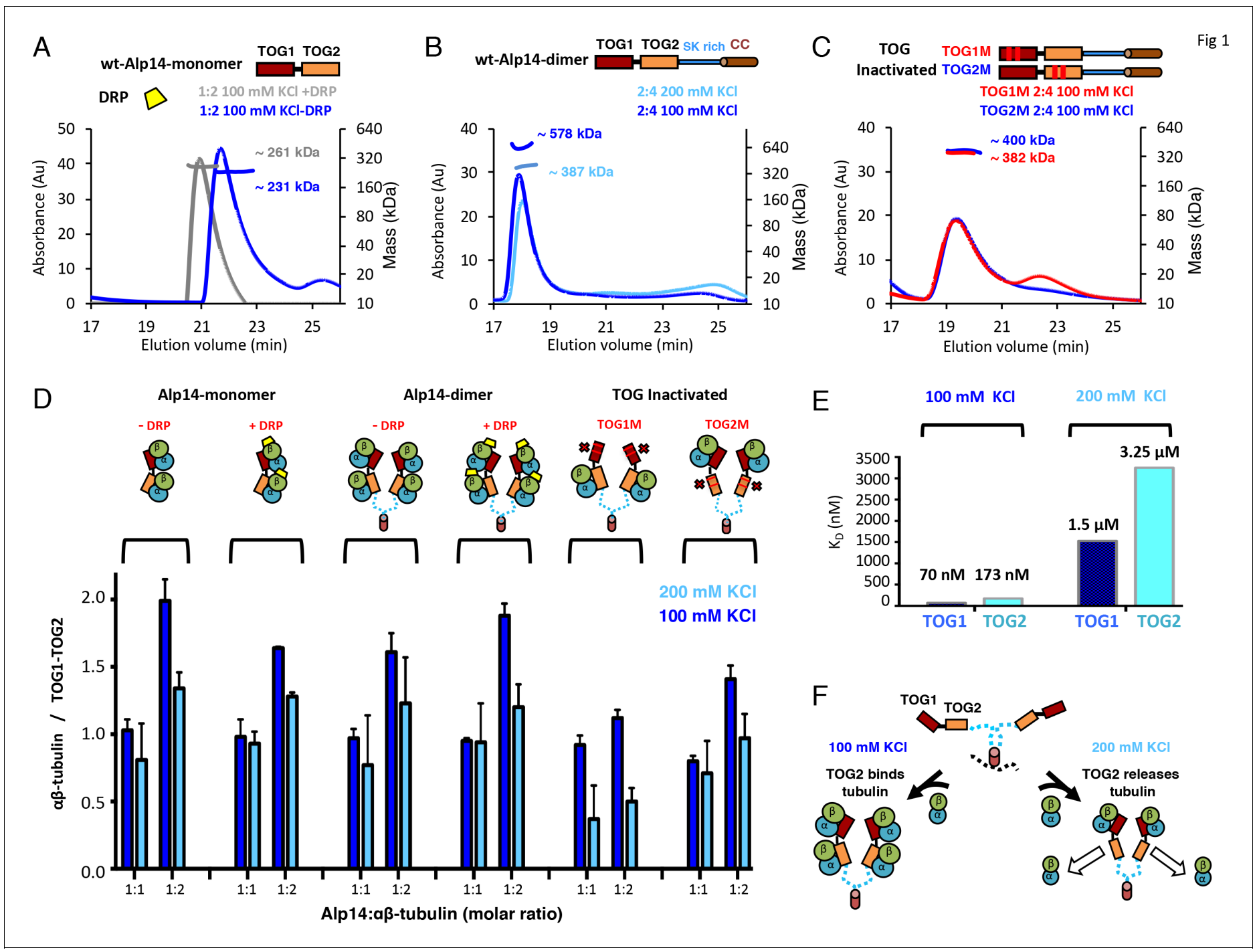

Figure 1. TOG1 and TOG2 domains bind $\alpha \beta$-tubulins and exchange them at different rates, within Alp14 TOG arrays. (A) Top, domain organization of Alp14-monomer. Bottom, SEC-MALS for wt-Alp14-monomer at $100 \mathrm{mM} \mathrm{KCl}$ with and without DRP, revealing two $\alpha \beta$-tubulins bound in a nonpolymerized state. (B) Top, domain organization of Alp14-dimer. Bottom, SEC-MALS for wt-Alp14-dimer in $\alpha \beta$-tubulin complex at 2:4 stoichiometry at $100 \mathrm{mM}$ and $200 \mathrm{mM} \mathrm{KCl}$, revealing that four $\alpha \beta$-tubulins bind dimeric-Alp14 with four TOG domains and two $\alpha \beta$-tubulins dissociate upon increase of ionic strength (masses reported in Table 2). (C) Top, organization of TOG-inactivated Alp14-dimer (C: TOG1M, TOG2M). Bottom, SEC-MALS of 4:2 TOG1M- and TOG2M- $\alpha \beta$-tubulin at $100 \mathrm{mM} \mathrm{KCl}$ revealing half of the $\alpha \beta$-tubulin binding stoichiometry compared to wt-Alp14-dimer (shown in B). (D) SEC-based titration measured through analyses of masses from SDS-PAGE (Figure 1-figure supplement 1; Materials and methods) of Alp14monomer, Alp14-dimer, and TOG1M and TOG2M with and without DRP binding, reveals the binding capacities of TOG1 and TOG2 domains in these constructs and their non-equivalence in $\alpha \beta$-tubulin exchange at $200 \mathrm{mM} \mathrm{KCl}$. DRP binding to $\alpha \beta$-tubulin does not influence $\alpha \beta$-tubulin binding to TOG arrays. Details are described in Figure 1-figure supplement 1-2. (E) Isothermal titration calorimetery (ITC) reveals TOG1 and TOG2 exchange $\alpha \beta-$ tubulin with non-equivalent rates at $200 \mathrm{mM} \mathrm{KCl}$. At $100 \mathrm{mM} \mathrm{KCl}$, TOG1 and TOG2 both slowly dissociate from $\alpha \beta$-tubulin with $\mathrm{K}_{\mathrm{D}}=70 \mathrm{nM}$ and $K_{D}=173 \mathrm{nM}$, respectively. At $200 \mathrm{mM} \mathrm{KCl}$, TOG1 dissociates from $\alpha \beta$-tubulin slowly with a $K_{D}=1.50 \mu \mathrm{M}$ while TOG2 mostly dissociates from $\alpha \beta$ tubulin with a $K_{D}=3.25 \mu \mathrm{M}$. ITC binding curves are shown in Figure 1-figure supplement 3. (F) Model for non-equivalent activities of TOG1 and TOG2 within TOG array for recruiting $\alpha \beta$-tubulins.

DOI: https://doi.org/10.7554/eLife.38922.002

The following figure supplements are available for figure 1 :

Figure supplement 1. The Alp14 TOG array: $\alpha \beta$-tubulin binding capacities reveal non-equivalent behavior of TOG1 and TOG2 in different conditions. DOI: https://doi.org/10.7554/eLife.38922.003

Figure supplement 2. Molar ratios of Alp14 constructs binding to $\alpha \beta$-tubulin binding in the presence of Darpin-D1 (DRP) show no change in stoichiometry, and control SEC-MALS traces. DOI: https://doi.org/10.7554/eLife.38922.004

Figure 1 continued on next page 
Figure 1 continued

Figure supplement 3. Isothermal titration calorimetry (ITC) reveals Alp14-TOG1 and TOG2 $\alpha \beta$-tubulin-binding affinities.

DOI: https://doi.org/10.7554/eLife.38922.005

measured at $100 \mathrm{mM} \mathrm{KCl}$. Together, our studies suggest that within the cellular $\alpha \beta$-tubulin concentration range $(5-10 \mu \mathrm{M})$ and $1 \mu \mathrm{M}$ Alp14 at $100 \mathrm{mM} \mathrm{KCl}$ or below, each TOG1-TOG2 subunit tightly binds two $\alpha \beta$-tubulins, whereas at $200 \mathrm{mM} \mathrm{KCl}$, each TOG1-TOG2 subunit binds one $\alpha \beta$-tubulin tightly via TOG1 and exchanges a second $\alpha \beta$-tubulin rapidly via TOG2. In conditions of $200 \mathrm{mM} \mathrm{KCl}$, $1 \mu \mathrm{M}$ Alp14, and 5-10 $\mu \mathrm{M} \alpha \beta$-tubulin, TOG1 domains is almost completely occupied by $\alpha \beta$-tubulin, while TOG2 domains is mostly dissociated from $\alpha \beta$-tubulin (Figure 1F).

\section{X-ray structures of a recruitment complex: pseudo-dimeric TOG1-TOG2 arrays in a head-to-tail square assembly that pre-orients $\alpha \beta$-tubulins}

Our biochemical analyses suggest that structural studies of TOG array: $\alpha \beta$-tubulin complexes must be conducted at lower ionic strengths of $80-100 \mathrm{mM} \mathrm{KCl}$ and at high $\alpha \beta$-tubulin concentrations to avoid $\alpha \beta$-tubulin dissociation from TOG2 domains. To further increase TOG1-TOG2: $\alpha \beta$-tubulin complex formation and inhibit $\alpha \beta$-tubulin self-assembly under such conditions, we utilized the designed ankyrin repeat protein (DARPin) D1 (herein termed DRP), which specifically binds and neutralizes the $\beta$-tubulin polymerizing interface (Pecqueur et al., 2012). First, we studied if DRP binding to $\alpha \beta$-tubulin influenced wt-Alp14-monomer or wt-Alp14-dimer binding stoichiometries to multiple $\alpha \beta$-tubulins. We measured binding molar ratios using quantitative-SEC and stoichiometry using SEC-MALs for DRP-bound- $\alpha \beta$-tubulin to wt-Alp14-dimer and wt-Alp14-monomer in $80-100 \mathrm{mM} \mathrm{KCl}$ conditions, respectively (Figure 1A; Figure 1-figure supplement 2A-F; Tables 1 and 2). We showed that DRP did not affect the simultaneous binding of multiple $\alpha \beta$-tubulins to TOG1-TOG2 arrays in either wtAlp14-monomer or wt-Alp14-dimer. $1 \mu \mathrm{M}$ wt-Alp14-dimer formed a complex with four $\alpha \beta$-tubulins and four DRPs at a molar ratio of 2:4:4 (Figure 1D; Figure 1-figure supplement 2D-F). We measured a mass of the wt-Alp14-monomer: $\alpha \beta$-tubulin:DRP complex by SEC-MALS that indicated a 1:2:2 stoichiometry complex in which each TOG1-TOG2 subunit bound two $\alpha \beta$-tubulins, each of which bound its own DRP (Figure 1A,D; Figure 1-figure supplement 2A-C,G; Table 2). The ability

Table 1. The stoichiometry for MT polymerases TOG1-TOG2 binding $\alpha \beta$-tubulin and DARPin (DRP)

\begin{tabular}{|c|c|c|c|c|}
\hline Protein complex & Expected Mass (kDa) & $\begin{array}{l}\text { SEC-MALS Measured } \\
\text { Mass } \\
\text { (kDa) }\end{array}$ & $\begin{array}{l}\text { SEC } \\
\text { elution } \\
\text { volume } \\
\text { (mL) }\end{array}$ & $\begin{array}{l}\text { Apparent } \\
\text { Mass (kDa) }\end{array}$ \\
\hline$\alpha \beta$-Tubulin & $100 \mathrm{kDa}$ & $98 \pm 0.323^{*}$ & 12.9 & 104 \\
\hline Alp14-dimer & $150 \mathrm{kDa}$ & $143 \pm 1.70$ & 10.0 & 675 \\
\hline 1 Alp14-dimer: 1 Tubulin 80 mM KCl & $350 \mathrm{kDa}$ & $387 \pm 2.74$ & 9.27 & 463 \\
\hline 1 Alp14-dimer: 2 Tubulin $80 \mathrm{mM} \mathrm{KCl}$ & $550 \mathrm{kDa}$ & $578 \pm 1.41$ & 8.98 & 784 \\
\hline 1 Alp14-dimer: 1Tubulin 200 mM KCl & $350 \mathrm{kDa}$ & $304 \pm 11.8$ & 9.22 & 693 \\
\hline 1 Alp14-dimer: 2 Tubulin 200 mM KCl & $350 \mathrm{kDa}$ & $392 \pm 9.66$ & 9.67 & 549 \\
\hline 1 Alp14-dimer-TOG1M: 1 Tubulin $80 \mathrm{mM} \mathrm{KCl}$ & $350 \mathrm{kDa}$ & $400 \pm 7.3$ & 10.13 & 433 \\
\hline 1 Alp14-dimer-TOG2M: 2 Tubulin $80 \mathrm{mM} \mathrm{KCl}$ & $350 \mathrm{kDa}$ & $382 \pm 14$ & 9.76 & 524 \\
\hline Alp14-monomer & $62 \mathrm{kDa}$ & $77.8 \pm 1.21$ & 12.98 & 99 \\
\hline 1 Alp14-monomer: 2 Tubulin $80 \mathrm{mM} \mathrm{KCl}$ & $262 \mathrm{kDa}$ & $264 \pm 1.31$ & 11.17 & 253 \\
\hline 1 Alp14-monomer: 2 Tubulin: 2 DRP $80 \mathrm{mM} \mathrm{KCl}$ & $298 \mathrm{kDa}$ & $312 \pm 2.32$ & 10.94 & 285 \\
\hline 1 Alp14-dimer-INT1: 2 Tubulin 80mM KCl & $550 \mathrm{kDa}$ & $533 \pm 3.11$ & 8.95 & 775 \\
\hline 1 Alp14-dimer-INT2: 2 Tubulin 80mM KCl & $550 \mathrm{kDa}$ & $580 \pm 3.11$ & 8.90 & 770 \\
\hline 1 Alp14-dimer-INT1+2: 2 Tubulin $80 \mathrm{mM} \mathrm{KCl}$ & $550 \mathrm{kDa}$ & $540 \pm 3.22$ & 8.80 & 790 \\
\hline
\end{tabular}

*Standard error is defined based on fitting data across peaks using Astra-software.

DOI: https://doi.org/10.7554/eLife.38922.006 
Table 2. Capacities of MT polymerase TOG1-TOG2 to bind $\alpha \beta$-tubulin, influenced by ionic strength

Protein Complex

(Alp14-monomer concentration 4.43 uM)

$\mu \mathrm{M}$ tubulin* bound

$\mu \mathrm{M}$ tubulin

TOG1-TOG2

wt-Alp14-monomer $(2 \mu \mathrm{M})$

1 Alp14-monomer: 1 Tub $100 \mathrm{mM} \mathrm{KCl}$

1 Alp14-monomer: 2 Tub $100 \mathrm{mM} \mathrm{KCl}$

$4.57 \pm 0.08$

$0.43 \pm 0.08$

: $\alpha \beta$-tubulin

1 Alp14-monomer: 1 Tub $200 \mathrm{mM} \mathrm{KCl}$

$8.82 \pm 0.16$

$1.18 \pm 0.16$

1.04

1 Alp14-monomer: 2 Tub $200 \mathrm{mM} \mathrm{KCl}$

$3.37 \pm 0.27$

$1.63 \pm 0.27$

2

1 Alp14-monomer:1 Tub:1 DRP $100 \mathrm{mM} \mathrm{KCl}$

$5.97 \pm 0.12$

$4.03 \pm 0.12$

0.76

1 Alp14-monomer:2 Tub:2 DRP $100 \mathrm{mM} \mathrm{KCl}$

$4.33 \pm 0.13$

$0.67 \pm 0.13$

1.35

Alp14-monomer:2 Tub:2 DRP $100 \mathrm{mM} \mathrm{KCl}$

$7.23 \pm 0.01$

$2.77 \pm 0.01$

1.64

1 Alp14-monomer:1 Tub:1 DRP $200 \mathrm{mM} \mathrm{KCl}$

$4.13 \pm 0.09$

$0.87 \pm 0.09$

1 Alp14-monomer: 2 Tub 2 DRP $200 \mathrm{mM} \mathrm{KCl}$

$5.67 \pm 0.03$

$4.34 \pm 0.03$

1.28

wt-Alp14-dimer $(1 \mu \mathrm{M})$

\begin{tabular}{|c|c|c|c|}
\hline 1 Alp14-dimer: 1 Tub 100 mM KCl & $4.31 \pm 0.07$ & $0.69 \pm 0.07$ & 0.98 \\
\hline 1 Alp14-dimer: 2 Tub 100 mM KCl & $7.11 \pm 0.14$ & $2.89 \pm 0.14$ & 1.61 \\
\hline 1 Alp14-dimer: 1 Tub 200 mM KCl & $3.44 \pm 0.37$ & $1.56 \pm 0.37$ & 0.78 \\
\hline 1 Alp14-dimer: 2 Tub 200 mM KCl & $5.46 \pm 0.34$ & $4.55 \pm 0.34$ & 1.24 \\
\hline 1 Alp14-dimer: 1 Tub: 2 DRP 100 mM KCl & $4.23 \pm 0.02$ & $0.78 \pm 0.02$ & 0.96 \\
\hline 1 Alp14-dimer: 1 Tub: 2 DRP 100 mM KCl & $8.33 \pm 0.09$ & $1.67 \pm 0.09$ & 1.89 \\
\hline 1 Alp14-dimer: 1 Tub: 1 DRP 200 mM KCl & $4.17 \pm 0.29$ & $0.83 \pm 0.29$ & 0.95 \\
\hline 1 Alp14-dimer: 2 Tub: 2 DRP 200 mM KCl & $6.02 \pm 0.17$ & $3.99 \pm 0.17$ & 1.36 \\
\hline \multicolumn{4}{|l|}{ TOG square Interface mutants $(1 \mu \mathrm{M})$} \\
\hline 1 INT1: 2 Tub $100 \mathrm{mM} \mathrm{KCl}$ & $8.34 \pm 0.07$ & $1.66 \pm 0.07$ & 1.74 \\
\hline 1 INT1: 2 Tub 200mM KCl & $5.67 \pm 0.04$ & $4.33 \pm 0.04$ & 1.18 \\
\hline 1 INT2: 2 Tub 100mM KCl & $9.52 \pm 0.3$ & $0.48 \pm 0.2$ & 2.02 \\
\hline 1 INT2: 2 Tub 200mM KCl & $6.82 \pm 0.2$ & $3.1 \pm 0.1$ & 1.45 \\
\hline 1 INT1+2: 2 Tub $100 \mathrm{mM} \mathrm{KCl}$ & $8.44 \pm 0.05$ & $1.56 \pm 0.05$ & 1.86 \\
\hline 1 INT1+2: 2 Tub $200 \mathrm{mM} \mathrm{KCl}$ & $6.85 \pm 0.04$ & $3.15 \pm 0.04$ & 1.46 \\
\hline \multicolumn{4}{|l|}{ Inactivated TOG mutants $(1 \mu \mathrm{M})$} \\
\hline 1 TOG1M: 1 Tub $100 \mathrm{mM} \mathrm{KCl}$ & $4.04 \pm 0.07$ & $0.97 \pm 0.07$ & 0.92 \\
\hline 1 TOG1M: 2 Tub $100 \mathrm{mM} \mathrm{KCl}$ & $4.94 \pm 0.06$ & $5.06 \pm 0.06$ & 1.12 \\
\hline 1 TOG1M: 1 Tub 200 mM KCl & $1.66 \pm 0.25$ & $3.34 \pm 0.25$ & 0.38 \\
\hline 1 TOG1M: 2 Tub $200 \mathrm{mM} \mathrm{KCl}$ & $2.23 \pm 0.1$ & $7.77 \pm 0.1$ & 0.51 \\
\hline 1 TOG2M: 1 Tub $100 \mathrm{mM} \mathrm{KCl}$ & $3.53 \pm 0.04$ & $1.48 \pm 0.04$ & 0.8 \\
\hline 1 TOG2M: 2 Tub $100 \mathrm{mM} \mathrm{KCl}$ & $6.24 \pm 0.10$ & $3.76 \pm 0.1$ & 1.41 \\
\hline 1 TOG2M: 1 Tub 200 mM KCl & $3.15 \pm 0.24$ & $1.85 \pm 0.24$ & 0.72 \\
\hline 1 TOG2M: 2 Tub 200 mM KCl & $4.28 \pm 0.18$ & $5.72 \pm 0.18$ & 0.97 \\
\hline
\end{tabular}

* Standard error is defined based on combined data from duplicated SEC runs.

DOI: https://doi.org/10.7554/eLife.38922.010

of Alp14-bound $\alpha \beta$-tubulin to bind stoichiometric amounts of DRP suggests that $\alpha \beta$-tubulins recruited by TOG arrays are in a non-polymerized state upon initial association with Alp14. This feature is consistent with a reported lack of cooperativity described between TOG1 and TOG2 in binding to $\alpha \beta$-tubulins (Ayaz et alo, 2014). Thus, we used this strategy to identify crystallization conditions using TOG array orthologs from a variety of organisms (see Materials and methods).

Crystals of the Saccharomyces kluyveri ortholog of Alp14 (herein termed sk-Alp14-monomer: residues 1-550) bound to $\alpha \beta$-tubulins and DRP grew in conditions similar to those used for SEC and SEC-MALS (Figure 2-figure supplement 1A). Using crystals with either a native sk-Alp14-monomer 
or an sk-Alp14-monomer with a modified TOG1-TOG2 linker sequence (termed sk-Alp14-monomerSL; see Materials and methods; Figure 2-figure supplement 2), we determined X-ray structures for 1:2:2 TOG1-TOG2 array: $\alpha \beta$-tubulin:DRP from complexes using sk-Alp14-monomer and sk-Alp14monomer-SL using molecular replacement (see Materials and methods) at $4.4 \AA$ and $3.6 \AA$ resolution, respectively (Table 3 and Figure 2-figure supplement 1B,C). In the structures, TOG1 domains were clearly differentiated from TOG2 domains by their conserved $\mathrm{C}$-terminal extension and jutting $\alpha$-helix that were unambiguously identified in density-modified maps (Figure 2-figure supplement 1D,E). Each asymmetric unit contained two wheel-shaped assemblies (Figure 2-figure supplement 1F) representing two sets of alternating TOG1 and TOG2 domains oriented in a square-like conformation (termed the TOG square), with each TOG domain binding a DRP-capped $\alpha \beta$-tubulin on its outer edge. Excluding the 10-residue TOG1-TOG2 linker region immediately

Table 3. X-ray Crystallographic and Refinement statistics of MT polymerase: $\alpha \beta$-tubulin:DRP.

\begin{tabular}{|c|c|c|c|c|}
\hline Data collection & $\begin{array}{l}\text { 1:2:2 sk-Alp14-monomer: } \\
\alpha \beta-T u b u l i n: D R P\end{array}$ & $\begin{array}{l}\text { 1:2:2 sk-Alp14-monomer-SL: } \\
\alpha \beta \text {-Tubulin:DRP }\end{array}$ & $\begin{array}{l}\text { 1:2:2 sk-Alp14-monomer: } \\
\alpha \beta-T u b u l i n: D R P \Delta N\end{array}$ & $\begin{array}{l}\text { 1:2:2 sk-Alp14-dimer: } \\
\alpha \beta \text {-Tubulin:DRP } \Delta N\end{array}$ \\
\hline Resolution range $(\AA)$ & $96.59-4.40(4.64-4.40)^{\star}$ & $59.45-3.60(3.79-3.60)^{\star}$ & $57.56-3.20(3.37-3.20)^{\star}$ & $99.83-3.5(3.69-3.50)^{\star}$ \\
\hline Space group & $P 2_{1}$ & $P 2_{1}$ & $P 2_{1}$ & $P 2_{1}$ \\
\hline Wavelength $(\AA)$ & 0.9792 & 0.9792 & 0.9792 & 0.9792 \\
\hline $\begin{array}{l}\text { Unit cell }(\AA): a, b, c \\
\left(^{\circ}\right): \beta\end{array}$ & $\begin{array}{l}218.80,107.65,282.74 \\
90.38\end{array}$ & $\begin{array}{l}218.48,106.15,282.23 \\
90.39\end{array}$ & $\begin{array}{l}\text { 115.13, 194.99, } 149.57 \\
90.19\end{array}$ & $\begin{array}{l}122.73199 .67,162.70 \\
90.09\end{array}$ \\
\hline Total number of observed reflections & 229567 & 380856 & 298551 & 235576 \\
\hline Unique reflections & $80099\{68039\}^{\dagger}$ & $142673\{121943\}^{\dagger}$ & $104265\{88337\}^{\dagger}$ & 91368 \\
\hline Average mosaicity & 0.57 & 0.38 & 0.64 & 0.50 \\
\hline Multiplicity & $2.9(2.9)^{*}$ & $2.7(2.7)^{*}$ & $2.9(2.9)^{*}$ & $2.6(2.4)^{*}$ \\
\hline Completeness (\%) & $95.4(94.8)\{80.6\}^{\dagger}$ & $95.0(96.7)\{79.0\}^{\dagger}$ & $96.2(97.9)\{82.0\}^{\dagger}$ & $92.9(90.2)^{\star}$ \\
\hline Wilson B-factor $\left(\AA^{2}\right)$ & 82.7 & 81.4 & 46.6 & - \\
\hline$<\mid / \sigma(I)>$ & $4.9(1.9)^{\star}$ & $4.8(1.2)^{*}$ & $5.8(1.5)^{\star}$ & $4.8(1.1)^{\star}$ \\
\hline$R_{\text {merge }}{ }^{c}$ & $0.14(0.48)^{*}$ & $0.13(0.65)^{*}$ & $0.13(0.65)^{*}$ & $0.14(0.63)^{*}$ \\
\hline \multicolumn{5}{|l|}{ Structure refinement } \\
\hline$R_{\text {work }}$ & $0.23(0.26)^{*}$ & $0.20(0.24)^{*}$ & $0.18(0.23)^{\star}$ & - \\
\hline$R_{\text {free }}$ & $0.26(0.33)^{\star}$ & $0.24(0.26)^{\star}$ & $0.24(0.26)^{\star}$ & - \\
\hline Complexes per asymmetric unit & 2 & 2 & 2 & - \\
\hline Number of atoms & 78030 & 77878 & 36865 & - \\
\hline Protein residues & 9989 & 9981 & 4661 & - \\
\hline Ligand atoms & 496 & 496 & 248 & - \\
\hline RMS bond lengths $(\AA)$ & 0.004 & 0.004 & 0.004 & - \\
\hline RMS bond angles $\left({ }^{\circ}\right)$ & 0.94 & 0.98 & 0.93 & - \\
\hline Ramachandran favored (\%) & 94.0 & 94.0 & 95.0 & - \\
\hline Ramachandran allowed (\%) & 5.4 & 5.5 & 4.5 & 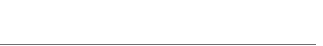 \\
\hline Ramachandran outliers (\%) & 0.3 & 0.3 & 0.2 & - \\
\hline Clashscore & 4.5 & 4.8 & 5.6 & - \\
\hline \multicolumn{5}{|l|}{ Mean $B$ values $\left(\AA^{2}\right)$} \\
\hline Overall & 108.4 & 98.3 & 48.6 & - \\
\hline Macromolecules & 108.6 & 98.4 & 48.6 & - \\
\hline Ligands & 78.5 & 91.5 & 36.4 & - \\
\hline
\end{tabular}

*Numbers represent the highest-resolution shell.

†Numbers represent the truncated data after treated with ellipsoidal truncation and anisotropic scaling

${ }^{\ddagger} R_{\text {merge }}=\Sigma_{h k} \Sigma_{i}\left|l_{i}(h k l)-l_{a v}(h k)\right| / \Sigma_{h k} \Sigma_{i} l_{j}(h k l)$.

DOI: https://doi.org/10.7554/eLife.38922.013 
preceding TOG2, the remaining 40 residues of the linker were disordered (Figure 2-figure supplement $1 \mathrm{~F}-\mathrm{I})$.

The dimension of each wheel-like assembly was $210 \times 198 \times 60 \AA$ (Figure 2A). The 2:4:4 stoichiometry observed in the X-ray structure matched the stoichiometry measured for wt-Alp14-dimer: $\alpha \beta$ tubulin:DRP complexes (Figure 1D; Figure 1-figure supplement 2D-F). We hypothesized that skAlp14-monomer formed dimeric organization, despite the lack of dimerization domains, due to the high concentration of these complexes during crystallization. X-ray structures revealed two TOG1TOG2 subunits in a pseudo-dimeric assembly forming the core of these complexes. In a TOG square, each TOG domain was bound to a curved $\alpha \beta$-tubulin capped by a DRP through its outward-facing binding interface and was not in contact with the neighboring TOG-bound $\alpha \beta$-tubulin (Figure 2A; Figure 2-figure supplement 1F-H). The distances and interaction patterns between residues of $\alpha$ tubulin and DRP bound to a neighboring $\beta$-tubulin indicated that DRP only interacts with its cognate $\beta$-tubulin and does not bind a neighboring $\alpha$-tubulin (Figure 2-figure supplement $1 \mathrm{~J}, \mathrm{~K}$ ). The latter suggests that DRP has no effect on stabilizing each TOG square assembly. Rather, DRP binding only caps $\beta$-tubulin, presenting a significant impediment to the polymerization of $\alpha \beta$-tubulins while bound to the TOG1-TOG2 subunits. The $\alpha \beta$-tubulins bound to the TOG square are positioned in a polarized orientation, due to the asymmetry in the TOG domain $\alpha \beta$-tubulin interface and pseudo-dimeric TOG1-TOG2 subunit interfaces within the TOG square (see below). The $\beta$-tubulin on a TOG1-bound $\alpha \beta$-tubulin is rotated approximately $90^{\circ}$ from its polymer-forming interface relative to the adjacent $\alpha$-tubulin on a TOG2-bound $\alpha \beta$-tubulin (Figure $2 B$ ).

\section{Two interfaces stabilize TOG1-TOG2 subunits into a TOG square assembly}

The X-ray structures revealed that each TOG square is a dimer of TOG1-TOG2 array subunits assembled head-to-tail from alternating TOG1 and TOG2 domains. TOG domains were aligned along their narrow edges, analogous to four links attached head-to-tail forming an asymmetric square-like complex with two edges slightly longer than their orthogonal edges (Figure 2C,D). Two contact sites, which we term interfaces 1 and 2, stabilize the TOG square. These interfaces are formed by interactions formed via inter-HEAT repeat loops of each TOG domain, which are located on the opposite edges from the $\alpha \beta$-tubulin-binding sites. Although TOG1 and TOG2 domains are each $60 \AA$ long, the TOG square assembly is slightly rectangular with $115 \AA$ by $98 \AA$ dimensions due to wider overlaps between TOG1 and TOG2 domains leading to $10 \AA$ stagger at interface 1 sites, in contrast to the direct end-on corner-like interface 2 sites. Both interfaces 1 and 2 are stabilized by hydrophobic packing and ionic interaction zones (Figure $2 \mathrm{E}-\mathrm{H}$ ). Interface 1 packs a $668 \AA^{2}$ surface area and positions the TOG1 $\mathrm{C}$-terminus at $90^{\circ}$ to a 10-residue segment of the TOG1-TOG2 linker and the N-terminus of TOG2. The TOG1-TOG2 linker sequence forms an extended polypeptide that critically bridges interactions between TOG1 inter-HEAT repeat $6 \alpha$-helix/inter-HEAT 5-6 loop segment and the TOG2 inter-HEAT repeat 1-2/2-3 loop segments (Figure 2E,F). Interface 2 packs a $290 \AA^{2}$ surface area and positions the TOG2 C-terminus at $90^{\circ}$ to the $\mathrm{N}$-terminus of TOG1 (Figure 2G,H). In interface 2, the TOG2 inter-HEAT repeat 4-5 loop interacts with the TOG1 inter-HEAT repeat 1-2/ HEAT1 $\alpha$-helix (Figure 2G). The residues forming interface 1 and interface 2 within TOG1, TOG2, and linker regions are either moderately or highly conserved (Figure 2F, H; Figure 2-figure supplement 2). The total buried surface area stabilizing two sets of interfaces 1 and 2 in a TOG square is $1930 \AA^{2}$, which is moderate in size and dispersed for such a large assembly. This finding suggests that this conformation may be meta-stable and that DRP binding and its inhibition of $\alpha \beta$-tubulin polymerization may stabilize this intermediate.

\section{Cysteine crosslinking and mass spectrometry reveal Alp14-dimer forms TOG square assembly interfaces in solution}

Next, we examined and chemically trapped the direct physical interactions between TOG1 and TOG2 interfaces observed in TOG square structures using cysteine mutagenesis and disulfide crosslinking. We generated mutants with specific cysteine pairs within the two sides of interface 1 (S180C, L304C) or interface 2 (S41C, E518C) in native dimeric sk-Alp14 (termed sk-Alp14-dimer; residues 1724) (Figure $3 A, B$ ). We tested whether these interfaces formed inter-subunit contacts in dimeric TOG array by crosslinking via disulfide oxidation. A $110 \mathrm{kDa}$ crosslinked species was observed in all 


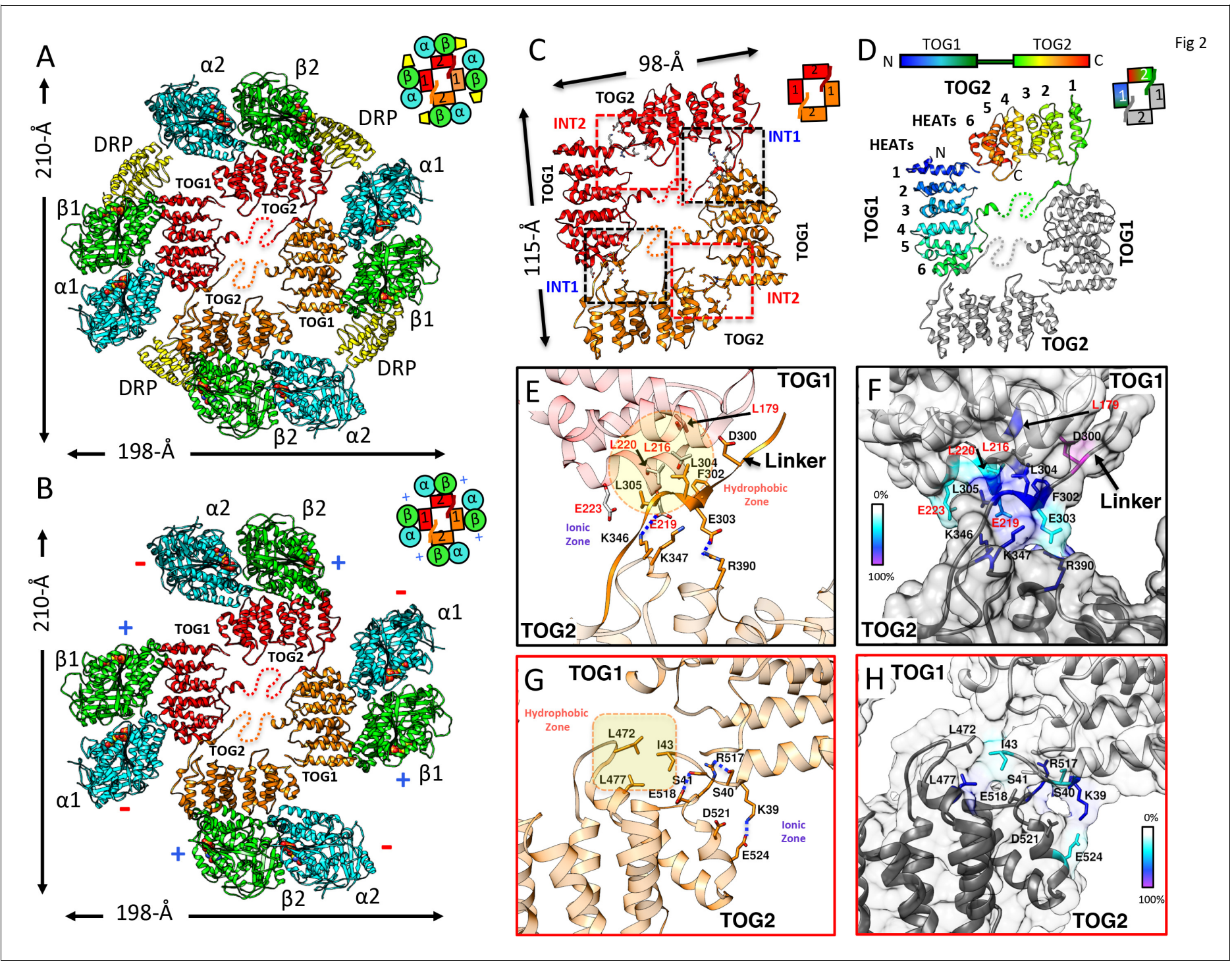

Figure 2. $X$-ray structures reveal $\alpha \beta$-tubulins bound in a wheel-like organization around a pseudo-dimeric TOG square complex. (A-B) $3.6 \AA$ X-ray crystal structure of the $S$. kluyveri 1:2:2 sk-Alp14: $\alpha \beta$-tubulin:DRP reveals pseudo-dimeric head-to-tail subunits (red and orange) in a TOG square assembly consisting of four TOG domains bound to four $\alpha \beta$-tubulins ( $\alpha$-tubulin shown in cyan and $\beta$-tubulin shown in green) in a wheel-like organization. (A) Structure with DRP (yellow) bound to each $\alpha \beta$-tubulin. (B) Structure with DRP computationally removed. Each $\alpha \beta$-tubulin ( $\alpha 1 \beta 1$ ) is positioned $90^{\circ}$ rotated from its polymer-forming interface on its neighboring $\alpha \beta$-tubulin ( $\left.\alpha 2 \beta 2\right)$. (C) Pseudo-dimeric TOG1-TOG2 subunits, shown in orange and red, respectively, form a head-to-tail TOG square (inset). Interface 1 is formed by the N-terminus of TOG2 and the TOG1-TOG2 linker binding to the C-terminus of the TOG1 domain of a second subunit, forming a $90^{\circ}$ corner. Interface 2 is formed by the $\mathrm{N}$-terminus of TOG 1 binding the C-terminus of TOG2 within the same subunit in a $90^{\circ}$ corner (Figure 2-figure supplement 11). (D) Rainbow view of TOG1-TOG2 with N-and C-termini displayed in a blue-to-red color gradient, while the other subunit is displayed in grey. Each TOG is composed of six HEAT repeats (numbered). (E) Close-up view of interface 1. A hydrophobic zone stabilizes interface1 (yellow and highlighted by red outline) involving Leu220 (L220) and Leu217 (L217) of the TOG1 inter-HEAT 5-6 loop, Leu179 (L179) of the HEAT 6 A-helix in TOG1 (red ribbon) stabilized by linker residues (solid orange) Phe302 (F302), Leu304 (L304), and Leu305 (L305). An ionic zone guides interface 1 involving Glu219 (E219) of TOG1 inter-HEAT 5-6 loop and Glu305 (E305) of the TOG1-TOG2 linker, forming salt bridges with Lys346 (K346) and Lys347 (K347) of the TOG2 (light orange) inter-HEAT 1-2 loop and Arg390 (R390) of the TOG2 HEAT 2,3 loop, respectively. (F) Close-up view of interface 1, as in C, displaying residue conservation based on the alignment shown in

Figure 2-figure supplement 2. (G) Close-up view of interface 2. A hydrophobic zone stabilizes interface 1 involving Leu477 (L477) and Leu472 (L472) of the TOG2 inter-HEAT4-5 loop with lle43 of the TOG1 inter-HEAT1-2 loop. lonic zone selectively guides interface 2, involving Lys39 (K39) and Ser41 (S41) of the TOG1 inter-HEAT1-2 loop and helix 1B with Arg517 (R517), Glu518 (E518), Asp521 (D521), and Glu524 (E524) of the TOG2 inter-HEAT5-6 loop and A-helix. (H) Close-up view of interface 2, as in D, displaying reside conservation based on the alignment in Figure 2-figure supplement 2. DOl: https://doi.org/10.7554/eLife.38922.007

The following figure supplements are available for figure 2 :

Figure 2 continued on next page 
Figure 2 continued

Figure supplement 1. X-ray crystallography and structure determination of 2:4:4 Alp14-monomer: $\alpha \beta$-tubulin:DRP complexes. DOI: https://doi.org/10.7554/eLife.38922.008

Figure supplement 2. Sequence conservation in TOG square interfaces across each TOG1 and TOG2 domain. DOI: https://doi.org/10.7554/eLife.38922.009

conditions where soluble $\alpha \beta$-tubulin was added, and mass spectrometry (LC/MS-MS) confirmed that this intermediate was indeed a crosslinked $\alpha$ - and $\beta$-tubulin heterodimer (Figure 3C,D; Figure 3figure supplement 1A). We also observed a $170 \mathrm{kDa}$ species that specifically formed in the $\alpha \beta$ tubulin-bound sk-Alp14 S180C-L304C mutant and not in the native sk-Alp14-dimer or the sk-Alp14S41C-E518C mutants. Furthermore, this $170 \mathrm{kDa}$ intermediate was also not observed with sk-Alp14S180C-L304C without $\alpha \beta$-tubulin (Figure 3D). Mass spectrometry confirmed that this $170 \mathrm{kDa}$ intermediate was indeed the sk-Alp14-S180C-L304C protein. Next, we mapped the cysteine residues involved in disulfide bonds in sk-Alp14-S180C-L304C mutants through peptide disulfide mapping after differential alkylation and mass spectrometry (see Materials and methods). This approach revealed only two classes of peptides in sk-Alp14-S180C-L304C with $105 \mathrm{Da}$ of mass added onto the cysteines, suggesting that they were engaged in disulfide bonds with the following sequence boundaries: 297-320 and 179-189 (Figure 3-figure supplement 1B). These two peptide sequences represent TOG1 inter-HEAT-repeat and TOG1-TOG2 linker regions, both of which are involved in forming interface 1 in the $\mathrm{X}$-ray structures (Figure 2). All the remaining peptides with cysteine residues that were identified in sk-Alp14-S180C-L304C included 57 Da in added mass, suggesting that they were in the reduced form and did not form disulfide bridges. Thus, these data directly provide independent support of interface 1 of the TOG square conformation forming in sk-Alp14-dimer in solution outside of the crystallographic setting, and of indeed being the inter-subunit dimeric interface between two TOG-array subunits, as visualized in the crystal structures (Figure $3 \mathrm{~A}, \mathbf{B}$ ).

\section{Disrupting TOG square assembly interfaces destabilizes organization, but does not affect $\alpha \beta$-tubulin binding}

We explored the role of interfaces 1 and 2 in stabilizing TOG square assembly and their effect on the $\alpha \beta$-tubulin capacity of TOG arrays. We generated three Alp14-dimer mutants that harbored either partially or fully disrupted interface 1 and 2 sites (Figure 4A-D). We targeted disruption of salt bridges or hydrophobic zones in interfaces 1 and 2 by mutating conserved alanines, leucines, or glutamates (Figure 2E, G; Figure 4A-D). Charged residues were either replaced with alanines or residues of the opposite charge, and hydrophobic residues were replaced with charged residues to dissociate hydrophobic interactions (Figure 4A-D). Initially, we disrupted interfaces 1 and 2 using one-, two-, or three-residue mutations in wt-Alp14-dimer. However, these mutants showed substantial levels of TOG square assemblies as assessed by negative stain electron microscopy (EM) (data not shown). Thus, we aimed to fully disrupt interfaces 1 and 2 by using seven to eight residues per interface. We mutated 8 residues in wt-Alp14-dimer to disrupt interface 1 (termed INT1; Figure 4B), 7 residues in wt-Alp14-dimer to disrupt interface 2 (termed INT2; Figure 4C), or 15 residues to disrupt both interfaces 1 and 2 (termed INT1 +2; Figure 4D) (see Materials and methods).

We studied the $\alpha \beta$-tubulin-binding capacities and stoichiometries of INT1, INT2, and INT1 +2 compared to wt-Alp14-dimer using quantitative-SEC and SEC-MALS approaches, respectively, as described in Figure 1. INT1, INT2, and INT1 +2 mutants bound nearly identical quantities of $\alpha \beta$ tubulin to wt-Alp14-dimer (Figure 4E; Figure 4F, G,H; Figure 4-figure supplement 1). INT1, INT2, and INT1 +2 bound approximately four $\alpha \beta$-tubulins at $80 \mathrm{mM} \mathrm{KCl}$ as assessed by SEC-MALS (Tables 1 and 2; Figure 4F, G,H) and approximately half of the bound $\alpha \beta$-tubulin dissociated at 200 $\mathrm{mM} \mathrm{KCl}$ as quantitated by quantitative-SEC (Figure 4D; Figure 4-figure supplement 1D,E,F).

We next used negative stain EM and 2D-single particle image analyses to compare the conformations of four $\alpha \beta$-tubulin-bound wt-Alp14-dimer assemblies to the $\alpha \beta$-tubulin:INT1, INT2 and INT1 +2 mutant assemblies. Negative stain images showed that 4:2 wt-Alp14-dimer: $\alpha \beta$-tubulin complexes formed two types compact particle-like assemblies of either 15 or $19 \mathrm{~nm}$ diameter compact circular complexes (Figure 2) matched the general features previously described for yeast Stu2p-tubulin or XMAP215-tubulin we previously described (Al-Bassam et alı, 2006; Brouhard et al., 2008). 2Dimage class averages were compared via projection-matching to low resolution-filtered structural 
A

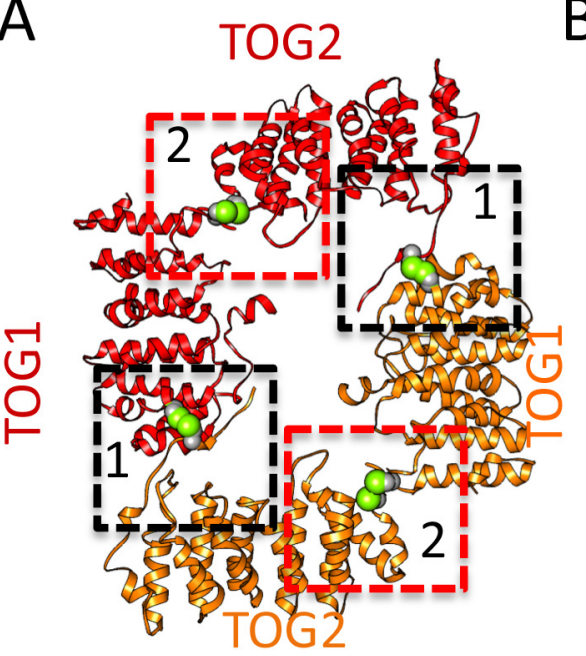

B Interface 1
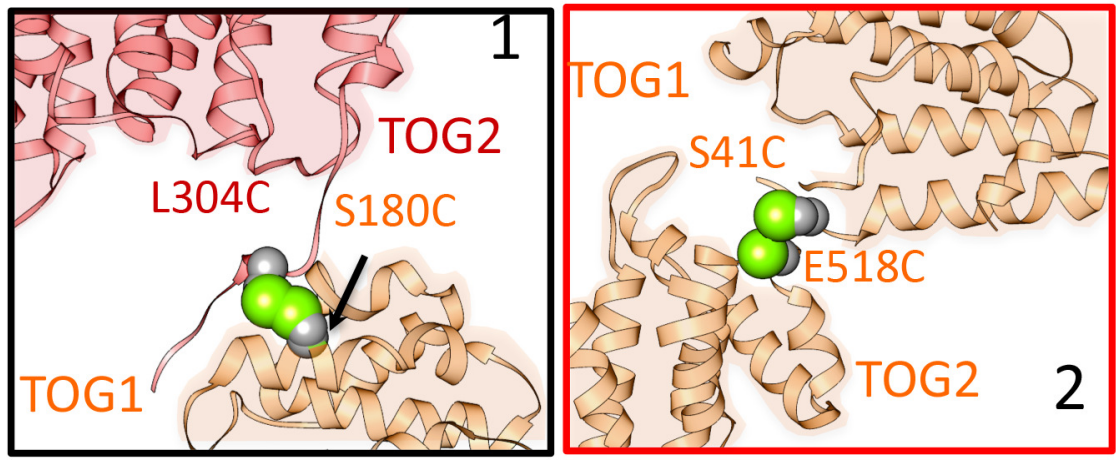

C

Sk-Alp14-dimer

D

Inter-subunit

Intra-subunit

WT

S180C L304C S41C E518C

Tubulin

\section{$\mathrm{S} 180 \mathrm{C}$}

S41C

L304C

E518C
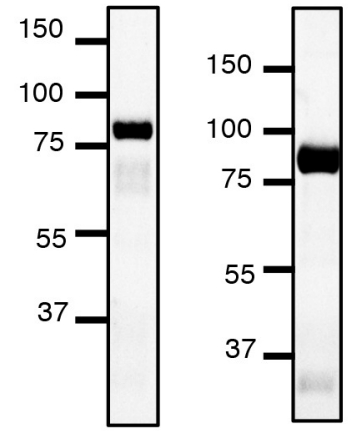
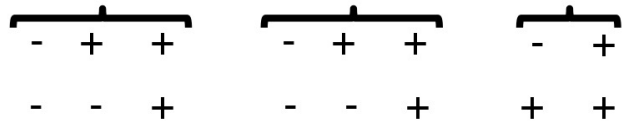
aß-tubulin - - +

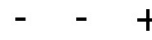

$+\quad+$

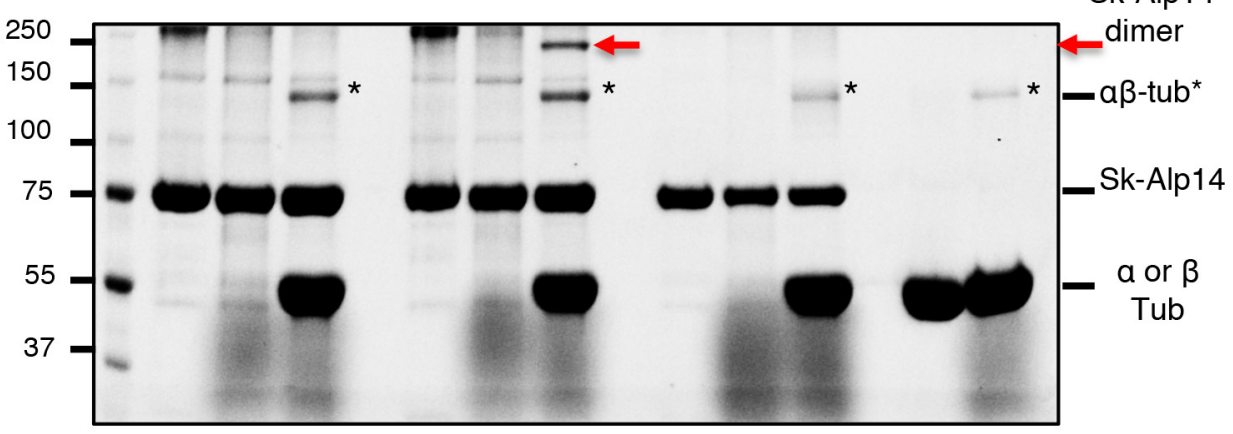

\section{E Disulfide peptide mapping with differential Cys-alkylation}

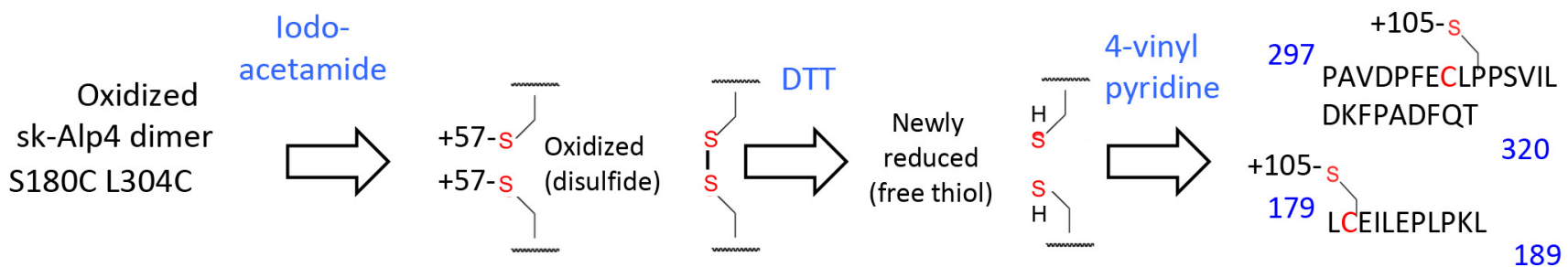

Figure 3. Cysteine mutagenesis/crosslinking and mass spectrometry-based peptide-mapping reveal that dimeric sk-Alp14 forms TOG square

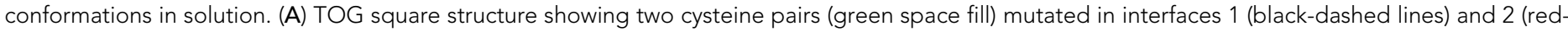

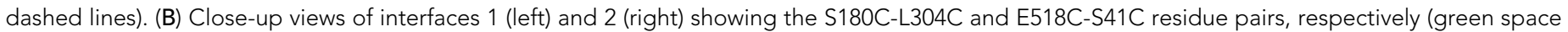

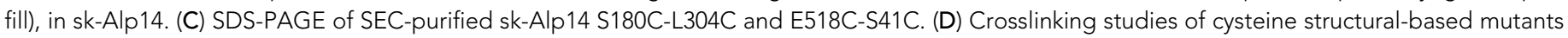

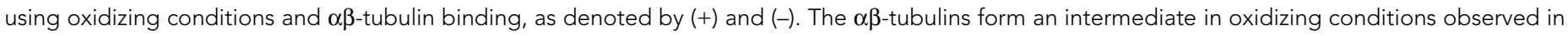
all conditions that include $\alpha \beta$-tubulin (marked ' ${ }^{\prime}$ ). S180C-L304C sk-Alp14 forms a dimeric 170 kDa intermediate upon oxidization and $\alpha \beta$-tubulin

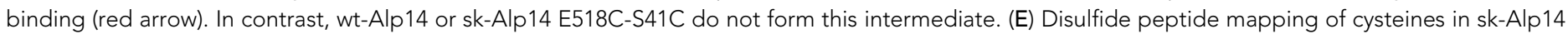
Figure 3 continued on next page 
Figure 3 continued

S180C-L304C using differential alkylation and LC/MS-MS. We used mass spectrometry (LC/MS-MS) after a differential alkylation strategy (Figure 3figure supplement 1D) to map peptides with disulfides. Briefly, oxidized sk-Alp14 S180C-L304C (170 kDa) SDS-PAGE-purified bands were subjected to proteolysis and treated with iodoacetamide. Under these conditions, $57 \mathrm{Da}$ in mass is added to peptides with reduced cysteines (free thiols), without affecting disulfides. Dithiothreitol was then used to reduce peptides with disulfides, which were then treated with 4-vinyl pyridine, which added 105 Da in mass to peptides with newly formed free thiols. Using LC/MS-MS, peptides with modified cysteines were identified based on added mass. Details provided in Figure 3-figure supplement 1D.

DOI: https://doi.org/10.7554/eLife.38922.011

The following figure supplement is available for figure 3 :

Figure supplement 1. Yeast dimeric TOG arrays form a TOG square assembly in solution as measured by cysteine crosslinking and mass spectrometry. DOI: https://doi.org/10.7554/eLife.38922.012

models for a TOG square with and without four $\alpha \beta$-tubulins, revealing TOG square bound to four $\alpha \beta$-tubulins matched well the density organization of the $19 \mathrm{~nm}$ diameter 2D-class averages (Figure 4I; Figure 4-figure supplement 2B). 2D-projections of a low-resolution filtered TOG square model without $\alpha \beta$-tubulins bound matched well the organization of the $15 \mathrm{~nm}$ diameter class averages suggesting those classes represent TOG squares that likely lost their bound $\alpha \beta$-tubulins on the grid (Figure 4I; Figure 4-figure supplement 2C). These data provide another line of support that TOG array subunits form square assemblies in solution, and that they match the organization of a TOG square bound to four $\alpha \beta$-tubulins (termed wheels) as observed in the crystal structure (Figure 2 ) or these dissociated from $\alpha \beta$-tubulin leading to isolated square-shaped assemblies (termed squares) (Figure 4). In contrast, INT1: $\alpha \beta$-tubulin complexes did not form square assemblies, and particles exhibited either open flexible organization with many inter-connected $8 \mathrm{~nm}$ long inter-connected densities or $16 \mathrm{~nm}$ long short filaments. 2D-image classification showed either $16 \mathrm{~nm}$ curved filaments, two $8 \mathrm{~nm}$ densities at right angles, or isolated $8 \mathrm{~nm}$ densities. 2D-projection matching using low resolution filtered models for single TOG1-TOG2 subunit bound to two $\alpha \beta$-tubulins from a TOG square structure, polymerized TOG- $\alpha \beta$-tubulin complexes (see next section), or with individual TOG- $\alpha \beta$-tubulin complexes (Ayaz et alo, 2012) matched well to the three types of class averages (Figure 4J; Figure 4-figure supplement 2D-F). These data confirmed that these complexes were indeed either single TOG1-TOG2 subunits, with 90 degree pre-arranged and non-polymerized $\alpha \beta$ tubulin assemblies, TOG1-TOG2 bound to two polymerized $\alpha \beta$-tubulins assemblies, or disordered assemblies composed of isolated TOG- $\alpha \beta$-tubulin complexes. INT2- $\alpha \beta$-tubulin complexes showed similar pattern of class averages that matched similar models as INT1- $\alpha \beta$-tubulin complexes suggesting similarly dissociation of a single interface in the TOG square (Figure $4 K$; Figure 4-figure supplement 2G-I). While, INT1 $+2 \alpha \beta$-tubulin complexes showed only dissociated, flexibly connected necklaces of $8 \mathrm{~nm}$ densities of $\alpha \beta$-tubulin (Figure $4 \mathrm{~L}$ ). 2D-image classification of these particles and 2D-projection matching of these complexes showed that each $8 \mathrm{~nm}$ class averages matched 2D-projections of a TOG-bound $\alpha \beta$-tubulin (Figure 4L; Figure 4-figure supplement $2 \mathrm{~J}-\mathrm{K}$ ). Our biochemical and negative stain-EM analyses suggest that wt-Alp14 dimer TOG arrays form square-shaped assemblies that match the organization observed in the TOG square crystal structure. Specific aspects of TOG square organization are clearly disrupted leading to the predicted defects in organization in the INT1, INT2, and INT1 +2 mutants without any effect on $\alpha \beta$-tubulin binding (Figure $4 \mathrm{~J}$, $K, L$ ). An interesting observation is that INT1 and INT2 mutants showed the propensity to form two polymerized $\alpha \beta$-tubulins filaments in some cases, suggesting that spontaneous in-solution $\alpha \beta$-tubulin polymerization occurs in the case of interface 1 and interface 2 destabilization (Figure $4 \mathrm{~J}$, K; Figure 4-figure supplement $2 \mathrm{E}, \mathrm{H}$ ). The dual inactivation of both TOG square interfaces in INT1 +2 resulted in dissociated TOG- $\alpha \beta$-tubulin complexes with a poor ability to form polymerize $\alpha \beta$-tubulins (Figure 4L; Figure 4-figure supplement 2).

\section{X-ray structure of a polymerization complex: TOG1-TOG2 subunit unfurling promotes the concerted polymerization of two $\alpha \beta$-tubulin}

The TOG square conformation shows how $\alpha \beta$-tubulins are recruited to TOG arrays but does not reveal how TOG arrays drive polymerization the recruited $\alpha \beta$-tubulins. We hypothesized that the TOG square structure may undergo a subsequent conformational change to promote polymerization of the recruited $\alpha \beta$-tubulins. To explore this transition, we created a biochemical approach to 


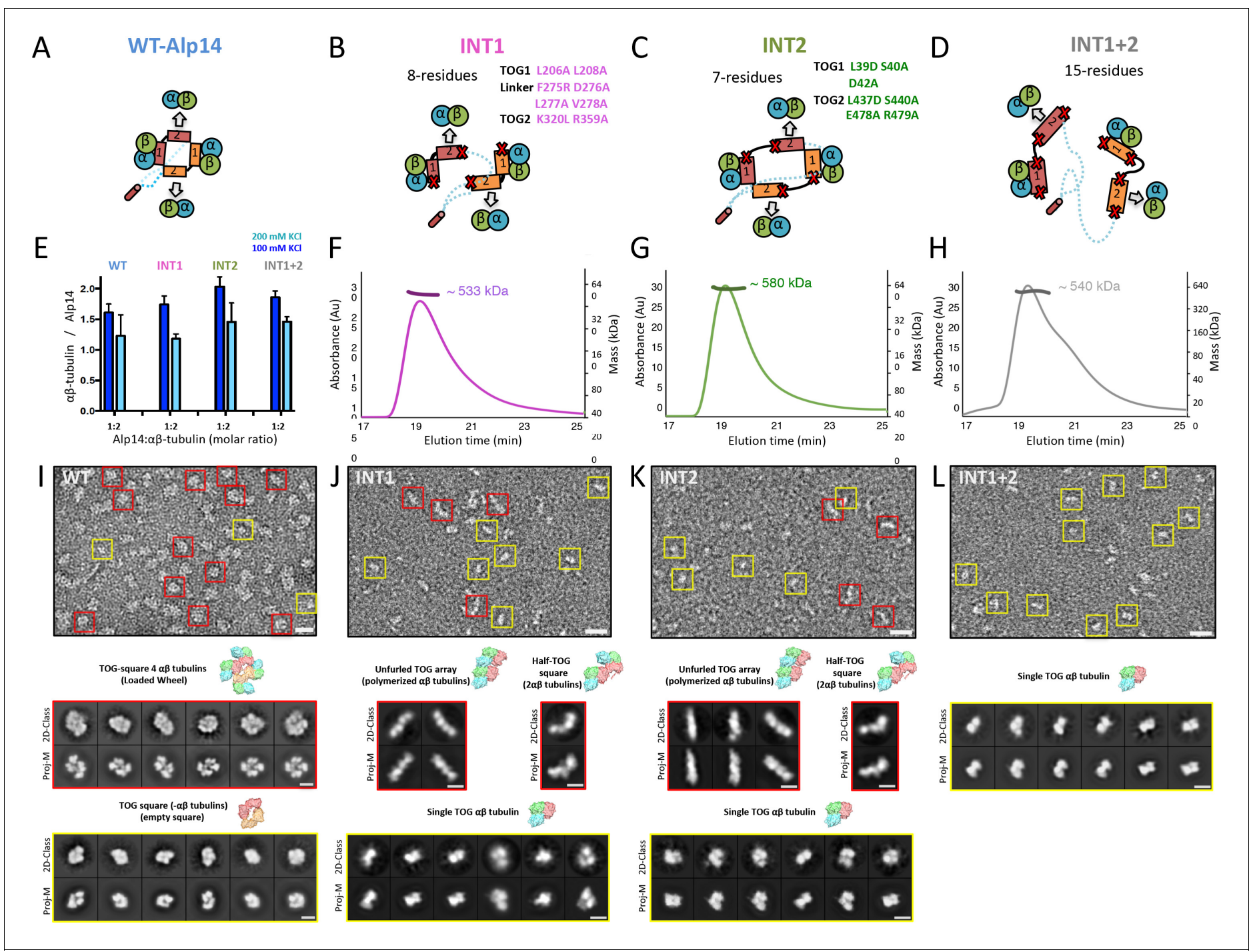

Figure 4. Inactivation of interfaces 1 and 2 destabilizes TOG square organization without affecting $\alpha \beta$-tubulin binding. (A-D) Generation of structurebased TOG square assembly-defective mutants using wt-Alp14-dimer (A) through inactivation of interface 1 in the INT1 mutant (B: INT1, pink; eight mutant residues), interface 2 in the INT2 mutant (C: INT2, green; seven mutant residues), or both interfaces 1 and 2 in the INT1 +2 mutant (D: INT1 +2, grey; 15 mutant residues). (E) Summary of SEC-measured $\alpha \beta$-tubulin-binding molar ratios of INT1, INT2, and INT1 +2 compared to wt-Alp14-dimer as described for Figure 1D suggests no defects in $\alpha \beta$-tubulin binding at $100 \mathrm{mM} \mathrm{KCl}$ (blue) and a similar decrease in $\alpha \beta$-tubulin binding upon $200 \mathrm{mM}$ $\mathrm{KCl}$ ionic strength increase (cyan). Additional information is described in Figure 4-figure supplement 1. (F-H) SEC-MALS of INT1: $\alpha \beta$-tubulin (F), INT2: $\alpha \beta$-tubulin (G), and INT1 +2 : $\alpha \beta$-tubulin $(\mathrm{H})$ complexes at 2:4 stoichiometry at $100 \mathrm{mM} \mathrm{KCl}$ (masses reported in Table 1). SEC-MALS reveals similar mass to wt-Alp14-dimer complexes with $\alpha \beta$-tubulin which are reported Table 2. (I) Top, raw negative stain EM image of wt-Alp14-dimer: $\alpha \beta$-tubulin at $100 \mathrm{mM} \mathrm{KCl}$ reveals wheel-shaped assemblies that are 15-19 nm in diameter as previously described for Stu2- $\alpha \beta$-tubulin complexes (Al-Bassam et al., 2006). Middle panel, $2 D$-classes reveal $19 \mathrm{~nm}$ wheel-shaped particles that match the $2 \mathrm{D}$-projection of $30 \AA$ resolution-filtered 2:4 TOG-square: $\alpha \beta$ tubulin complex (shown above the panel). These classes match the organization observed in the structure described in Figure 2. Bottom panel, second group of 2D-classes reveal small diamond-shaped particles that match the 2D-projection of a $30 \AA$ resolution-filtered model of the TOG square without $\alpha \beta$-tubulins (shown above the panel). ( $\mathrm{J}$ ) raw image of INT1: $\alpha \beta$-tubulin reveals elongated conformations with either bent-conformations composed of bent-particles with two $8 \mathrm{~nm}$ densities at 90 -degree angles or $16 \mathrm{~nm}$ filament-like particles. Middle panel, 2D-classes reveal $16 \mathrm{~nm}$ elongated classes that match the 2D-projection of either $30 \AA$ resolution-filtered TOG1-TOG2 bound to two polymerized tubulins (shown above the second row) as described structurally in the next section. Left panel, 90-degree bend class averages that match 2D-projections of a resolution-filtered model of one TOG1-TOG2 subunit of the TOG square bound to two tubulins at 90 degrees (as shown above). Bottom panel, 2D-classes reveal $8 \mathrm{~nm}$ lengths, which match 2D-projections of low-resolution-filtered TOG- $\alpha \beta$-tubulin complexes (PDB ID: 4FFB shown above the panel). (K) INT2 forms extended necklace shaped or extended $16 \mathrm{~nm}$ minifilaments. Middle panel, 2D-class averages reveal either $16 \mathrm{~nm}$ filament-like particles match 2D-projections of TOG1TOG2 bound to two polymerized $\alpha \beta$-tubulins (left) or bent particles that match 2D-projections of a bend TOG1-TOG2 subunit bound to two nonpolymerized $\alpha \beta$-tubulins, as observed in half a TOG square complex (right). (L) INT1 $+2: \alpha \beta$-tubulin complexes form only dissociated assemblies with Figure 4 continued on next page 
Figure 4 continued

randomly interconnected $8 \mathrm{~nm}$ assemblies. Bottom panel, 2D-classes reveal $8 \mathrm{~nm}$ lengths, which match 2D-projections of low-resolution-filtered TOG$\alpha \beta$-tubulin complexes (PDB ID:4FFB shown above the panel). Additional information can be found in Figure 4-figure supplement 1.

DOI: https://doi.org/10.7554/eLife.38922.014

The following figure supplements are available for figure 4:

Figure supplement 1. Inactivating interfaces 1 and 2 destabilizes the TOG square organization but does not influence $\alpha \beta$-tubulin-binding activity. DOI: https://doi.org/10.7554/eLife.38922.015

Figure supplement 2. Negative stain EM micrographs and class averages for the TOG square and its inactivated assemblies.

DOI: https://doi.org/10.7554/eLife.38922.016

partially release $\alpha \beta$-tubulin from polymerization by relieving DRP inhibition of $\alpha \beta$-tubulin polymerization while they are bound to TOG arrays. We reasoned that a structural transition may occur more readily if DRP dissociates from $\beta$-tubulin in a crystallization setting. We developed a strategy to conditionally release $\alpha \beta$-tubulin from polymerization while being recruited into TOG arrays by using a weakened affinity DRP. We reasoned that the increased dissociation of DRP may allow complexes to form polymerized $\alpha \beta$-tubulin intermediates in steady state, as seen in the negative stain studies. To accomplish this, we removed the $\mathrm{N}$-terminal ankyrin repeat of DRP (herein termed DRP $\Delta \mathrm{N}$ ). We measured DRP $\triangle \mathrm{N}$ affinity using ITC, revealing a three-fold decrease in its $\alpha \beta$-tubulin-binding affinity as compared to DRP (Figure 5A-B). During purification, complexes of 1:2:2 sk-Alp14-monomer: $\alpha \beta$ tubulin:DRP $\triangle \mathrm{N}$ behaved similarly to those assembled with DRP on SEC (Figure 5C; Figure 5-figure supplement 1). However, using crystallization conditions identical to those used to obtain the 4:4:2 TOG square conformation, we obtained crystals that exhibited a distinct rectangular morphology using sk-Alp14-monomer or sk-Alp14-dimer $\alpha \beta$-tubulin:DRP $\Delta \mathrm{N}$ complexes (see Materials and methods; Figure 5-figure supplement 2A). These crystals formed in the same space group (P2 $\left.{ }_{1}\right)$ with distinct unit cell dimensions compared to the TOG square crystals (Table 3). These crystals exclusively formed only when DRP $\Delta \mathrm{N}$ was used with $\alpha \beta$-tubulin:sk-Alp14-monomer or -dimer complexes. We determined an X-ray structure to $3.2 \AA$ resolution by molecular replacement using these crystals (Figure 5-figure supplement 2B). The structure revealed a novel assembly consisting of complexes with the stoichiometry of 1:2 sk-Alp14-monomer: $\alpha \beta$-tubulin and a single DRP $\Delta \mathrm{N}$ positioned on the top end of TOG2-bound $\beta$-tubulin (termed the 1:2:1 structure; Figure 5E,F; Figure 5-figure supplement 2C-D).

The refined 3.2 $\AA$ 1:2:1 X-ray structure revealed an extended conformation with two $\alpha \beta$-tubulins polymerized head-to-tail in a curved protofilament (Figure 5E-F). In this conformation, TOG1 and TOG2 did not form any interactions with each other and their adjoining linker became disordered (Figure 5E,F; Figure 5-figure supplement 2C,D). TOG1 and TOG2 were specifically bound to the lower and upper $\alpha \beta$-tubulins, respectively, of a highly curved protofilament. Only a single DRP $\Delta N$ capped the TOG2-bound $\alpha \beta$-tubulin (Figure 5E). Compared to the TOG square, this 'unfurling' rearrangement required $68^{\circ}$-rotation and $32 \AA$ translation of TOG2: $\alpha \beta$-tubulin hinging around interface 2 and TOG1- $\alpha \beta$-tubulin (Figure $6 \mathbf{A}, \mathbf{B}$ ). This transition promoted the concerted polymerization of TOG2: $\alpha \beta$-tubulin onto the plus-end of TOG1: $\alpha \beta$-tubulin, and consequently drove the dissociation of the second DRP $\triangle N$ (Figure $5 C_{,} D$ ). The two $\alpha \beta$-tubulin polymers in this complex were highly curved protofilaments $\left(16.4^{\circ}\right.$ inter-dimer interface). This protofilament structure displayed $\sim 3^{\circ}$ more curvature than RB3/stathmin- $\alpha \beta$-tubulin curved protofilament structures (Table 4; Figure 5-figure supplement 2E) (Nawrotek et al., 2011). Comparison of the $\alpha \beta$-tubulin dimer structure $(\alpha 2 \beta 2)$ within this $1: 2: 1$ structure to the unpolymerized $\alpha \beta$-tubulin structure revealed that polymerization is associated with a $5^{\circ}$ rotation and $10 \AA$ translation in the T7 loop and $\mathrm{H} 8$ helix of the TOG2-bound $\alpha$-tubulin, which engages TOG1-bound $\beta$-tubulin elements and the E-site-bound GDP nucleotide (Figure $6 C, D$ ). The latter conformational change likely stabilizes inter-dimer $\alpha \beta$-tubulin interfaces (Figure 5D), burying a $1650 \AA$ inter-dimer interface (Figure 5E,F). This conformational change occurs at a site similar to those observed during the MT lattice GTP hydrolysis transition (Alushin et al., 2014). Thus, the 1:2:1 unfurled structure represents a concerted $\alpha \beta$-tubulin postpolymerization intermediate promoted by a single TOG1-TOG2 subunit prior to straightening the protofilament during polymerization at MT plus-ends. 
A DRP $\alpha \beta$-tubulin affinity
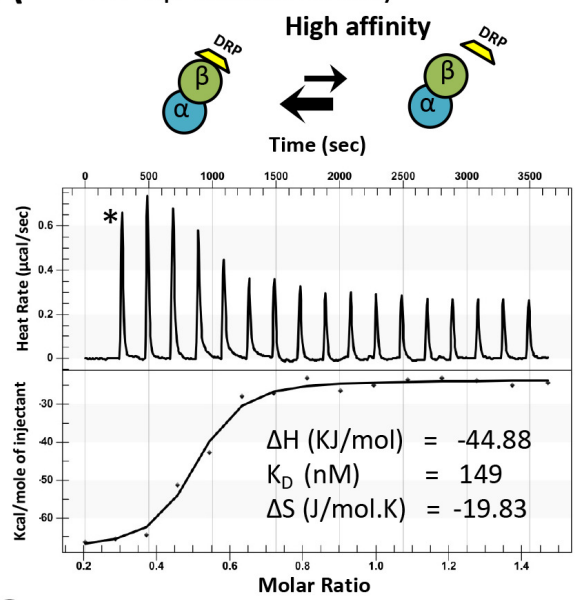

C

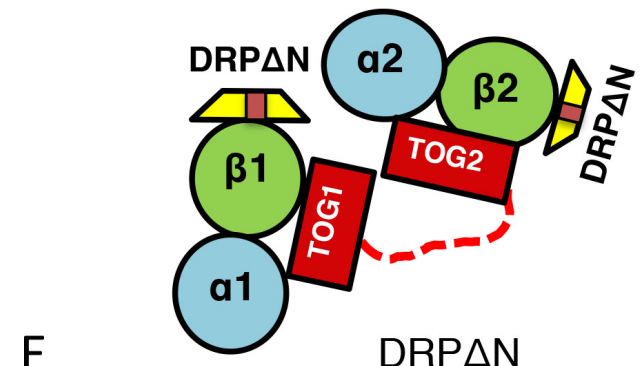

$E$

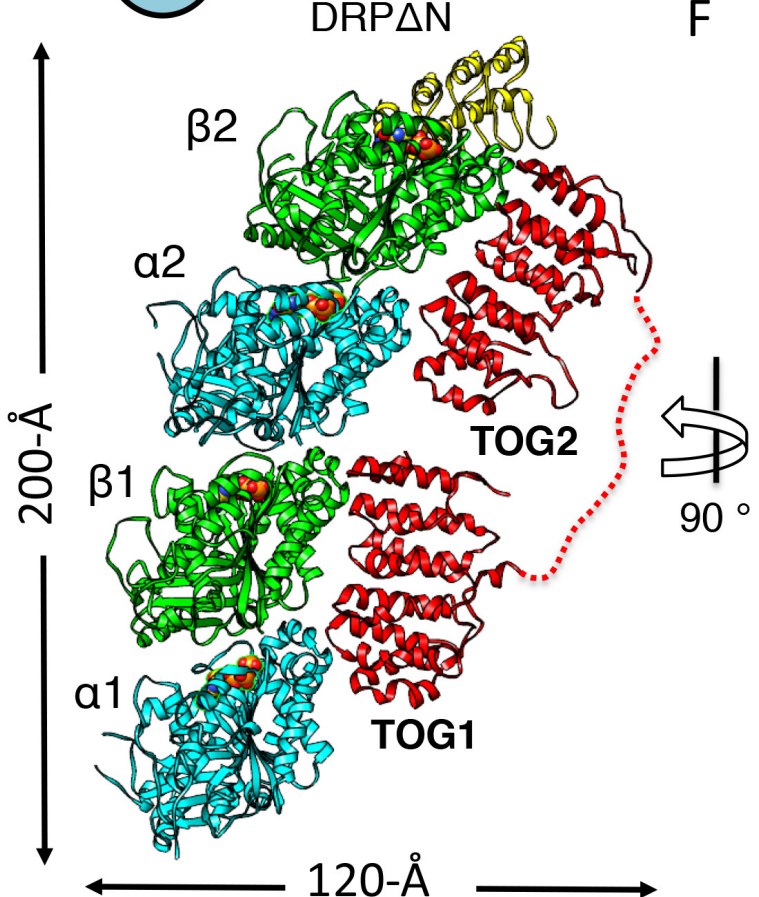

B DRP $\triangle N \alpha \beta$-tubulin affinity
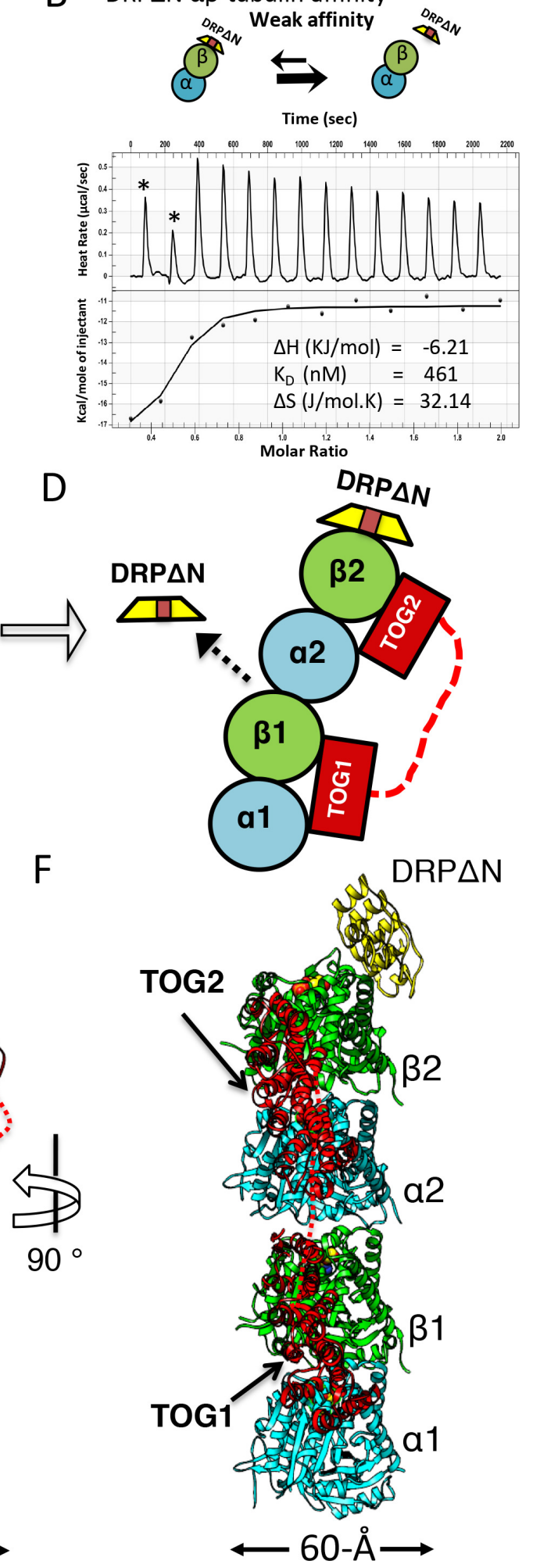

Figure 5. X-ray structure of 1:2:1 TOG-array: $\alpha \beta$-tubulin:DRP $\Delta N$ reveals unfurled TOG1-TOG2 array bound to two polymerized $\alpha \beta$-tubulins. (A, B) Top, schemes of DRP and DRP $\Delta N$ binding to $\alpha \beta$-tubulin. DRP shifts the equilibrium toward dissociation from $\alpha \beta$-tubulin. Bottom, isothermal titration calorimetery studies reveal a three-fold decrease in affinity of DRP $\Delta N$ binding to $\alpha \beta$-tubulin (461 nM) compared to DRP (149 nM). (C, D) Two schematic views of the TOG1-TOG2 $\alpha \beta$-tubulin complex transition from the TOG square (only half is shown) to the unfurled conformation upon DRP $\Delta N$

Figure 5 continued on next page 
Figure 5 continued

dissociation. (E, F) Two orthogonal views of the $3.2 \AA$ X-ray structure of 1:2:1 sk-Alp14 (red): $\alpha \beta$-tubulins (cyan and green):DRP $\Delta N$ (yellow) complex, indicating a polymerized protofilament state. TOG2 and TOG1 are bound to the upper ( $\alpha 2 \beta 2)$ tubulin and lower ( $\alpha 1 \beta 1)$ tubulin, respectively. DOI: https://doi.org/10.7554/eLife.38922.018

The following figure supplements are available for figure 5 :

Figure supplement 1. Strategy to promote $\alpha \beta$-tubulin polymerization using $D R P \Delta N$ and the structural comparison of DRP and DRP $\Delta N$ interfaces with $\alpha \beta$-tubulin.

DOl: https://doi.org/10.7554/eLife.38922.019

Figure supplement 2. X-ray crystallographic structure determination of 1:2:1 Sk-Alp14-monomer: $\alpha \beta$-tubulin:DRP $\Delta \mathrm{N}$ complex.

\section{Modeling $\alpha \beta$-tubulin-bound TOG square and unfurled structure docking onto microtubule plus-ends}

We next evaluated how X-ray structures of $\alpha \beta$-tubulin-bound TOG squares and unfurled TOG1TOG2 $\alpha \beta$-tubulin assemblies can dock onto protofilament tips at MT plus-ends. Atomic models for these states were overlaid onto the terminal $\alpha \beta$-tubulins of curved GTP or GDP $\alpha \beta$-tubulin protofilament models (Figure 7). Attempts to dock the $\alpha \beta$-tubulin onto protofilament ends exposed at the MT minus-end caused substantial steric clashes, supporting the notion that TOG square states are completely incompatible with docking at MT minus-ends (data not shown). The four $\alpha \beta$-tubulinbound TOG square assembly X-ray structure (Figure 2) was superimposed onto that of the terminal $\alpha \beta$-tubulin at protofilament ends in two docking orientations, either via the $\alpha \beta$-tubulins bound onto TOG1 or TOG2 (Figure 7A,B). We observed a slight steric surface overlap between the four $\alpha \beta$ tubulin-loaded TOG square and the curved protofilament when TOG1- $\alpha \beta$-tubulin was docked onto the $\beta$-tubulin at the protofilament plus-end (Figure 7A). This steric overlap was caused by overlap between $\alpha \beta$-tubulin-TOG2 from the second TOG1-TOG2 subunit in the TOG square with penultimate $\alpha \beta$-tubulin from the protofilament end (Figure 7A; Figure 7-figure supplement 1A). In contrast, we observed no steric contact when the TOG square was docked via $\alpha \beta$-tubulin-TOG2. In this orientation, the TOG1- $\alpha \beta$-tubulin from the second subunit was retracted by $10 \AA$ from the penultimate $\alpha \beta$-tubulin in the protofilament in contrast to the TOG1- $\alpha \beta$-tubulin docking (Figure 7B). The differences between steric overlap of the TOG square with the protofilament in these two docking orientations were due to the asymmetric dimensions of the TOG square, caused by stagger between TOG1 and TOG2 domains at interface 1 compared to interface 2 . These differences suggest that the destabilization of the TOG squares is more likely if TOG1- $\alpha \beta$-tubulin docks onto the protofilament plus-end in contrast to TOG2- $\alpha \beta$-tubulin docking. The unfurled 1:2 TOG1-TOG2: $\alpha \beta$-tubulin assembly can only be docked using TOG1- $\alpha \beta$-tubulin onto the protofilament plus-end and suggests that TOG2: $\alpha \beta$-tubulin is positioned the furthest away from the MT plus-end in this conformation. These models were used to assemble steps for a new MT polymerase model described in the discussion (Figure 8).

\section{Discussion}

\section{A 'polarized unfurling' model for TOG arrays as MT polymerases}

Using the combination of structural and biochemical analyses, we propose a new model for TOG arrays as MT polymerases, which we term the 'polarized-unfurling' model. The model is summarized in Figure 8 and animated in Video 1. This model is supported by X-ray structures of two states, negative EM studies of $\alpha \beta$-tubulin complexes of wt-Alp14-dimer and three interface inactivated Alp14 mutants, differences in affinities of TOG1 and TOG2 domains for $\alpha \beta$-tubulins, and described via docking of models at protofilament ends at each step (Figure 7). Our model suggests that TOG arrays form two separate conformations that together promote MT polymerase activity: an $\alpha \beta$-tubulin recruitment complex as denoted by the TOG square (Figures 2-4) and an unfurled MT plus-end polymerization complex denoted by the polymerized 1:2:1 TOG1-TOG2: $\alpha \beta$-tubulin X-ray structures (Figure 5). We have effectively trapped these states by regulating the polymerization propensities for $\alpha \beta$-tubulins using DRP affinity while bound to TOG arrays (Figures 2 and 5). We hypothesize that the association of the $\alpha \beta$-tubulin-bound TOG square onto the MT plus-ends, via $\beta$-tubulin binding, 
A

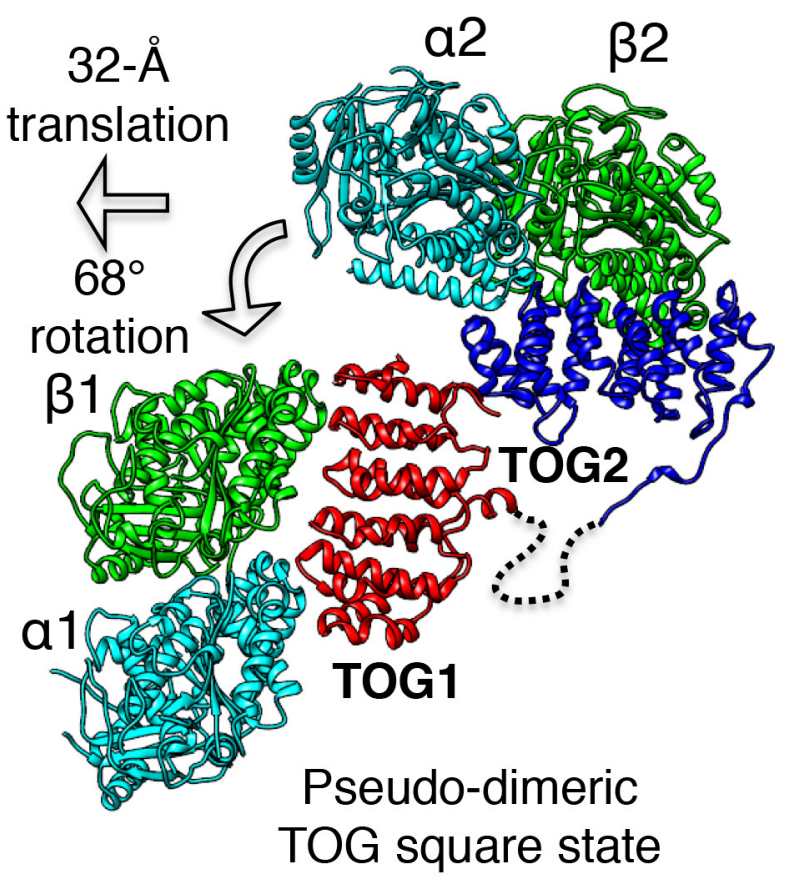

B

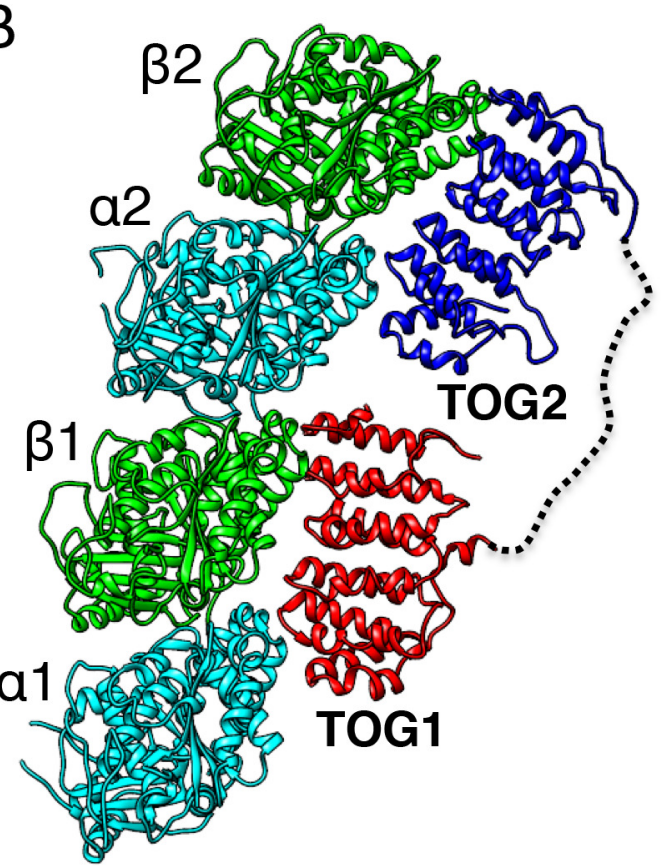

Unfurled Polymerized state

C

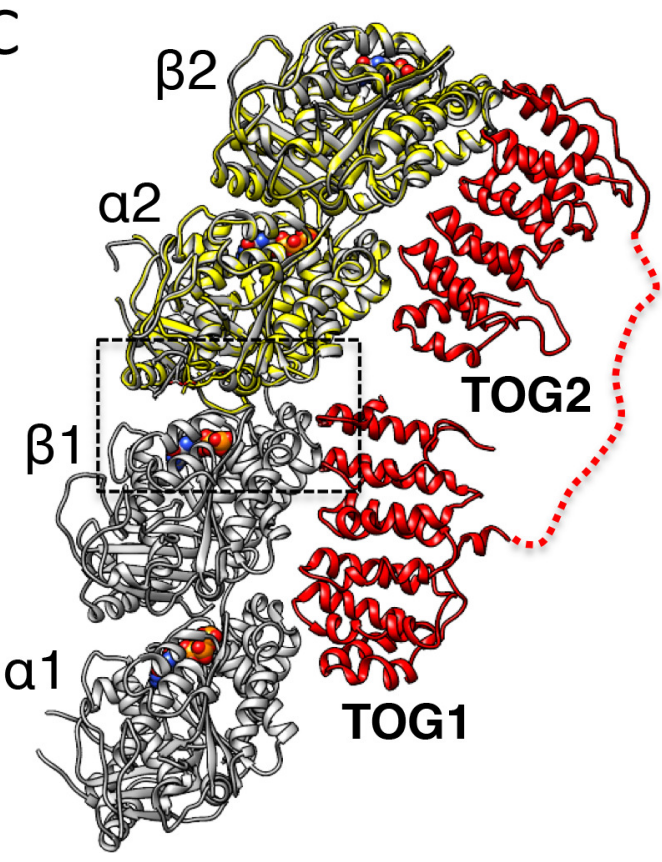

D
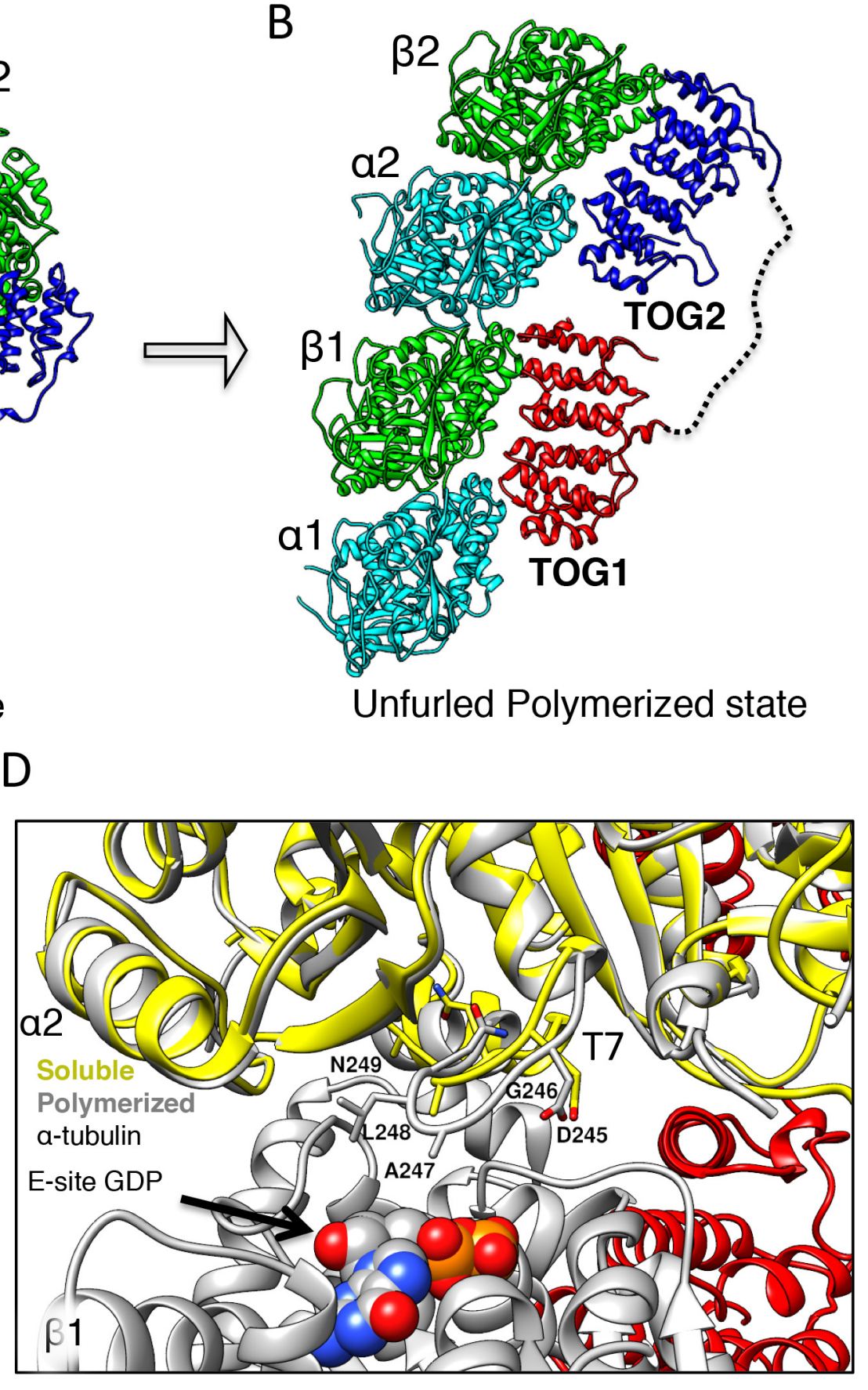

Figure 6. Unfurling the TOG array: TOG2 rotation around TOG1 promotes the bound $\alpha \beta$-tubulins to polymerize. (A and B) Conformational change of TOG2 (blue) around TOG1 (red) while each is bound to $\alpha \beta$-tubulin (green and cyan) from a corner subunit in the wheel assembly (left) and in the extended conformation (right). TOG2 rotates $32^{\circ}$ and translates $68 \AA$ upon release to drive $\alpha \beta$-tubulin polymerization into a protofilament. (C) Superimposing unpolymerized $\alpha \beta$-tubulin (yellow) onto the $\alpha 2 \beta 2$-tubulin shows a conformational change in $\alpha$-tubulin at the inter-dimer interface induced by polymerization. (D) Close-up view of the polymerized inter-dimer interface. Unpolymerized $\alpha \beta$-tubulin (yellow) is superimposed onto $\alpha 2 \beta 2$ (grey) of 1:2:1 structure. The $\alpha 2$-tubulin T7 loop and H8 helix engage the $\beta 1$-tubulin GDP nucleotide through a T7 loop $5 \AA$ translation and H8 helix $5^{\circ}$ rotation involving residues Asp245 (D245), Gly246 (G246), Ala247 (A247), and Leu248 (L248). 
Table 4. Buried Surface Area between $\alpha \beta$-tubulin dimer and TOG domains or DRP.

\begin{tabular}{|c|c|c|c|}
\hline Interface & $\begin{array}{l}\text { 1:2:2: sk-Alp14-monomer: } \\
\alpha \beta \text {-Tubulin: } \operatorname{DRP}\left(\AA^{2}\right)^{\star}\end{array}$ & $\begin{array}{l}\text { 1:2:2: sk-Alp14-monomer-SL: } \\
\alpha \beta \text {-Tubulin: DRP }\left(\AA^{2}\right)\end{array}$ & $\begin{array}{l}\text { 1:2:1: sk-Alp14-monomer: } \\
\alpha \beta-T u b u l i n: \text { DRP- } \Delta N\left(\AA^{2}\right)\end{array}$ \\
\hline TOG1 and TOG2-interface 2 & $273 \pm 35$ & $257 \pm 42$ & - \\
\hline TOG1-TOG2 dimer-interface 1 & $661 \pm 27$ & $702 \pm 18$ & - \\
\hline$\alpha \beta$-tubulin and TOG1 & $752 \pm 12$ & $786 \pm 42$ & $804 \pm 24$ \\
\hline$\alpha \beta$-tubulin and TOG2 & $916 \pm 23$ & $890 \pm 22$ & $863 \pm 12$ \\
\hline$\beta$-tubulin and DRP or DRP- $\Delta \mathrm{N}$ & $842 \pm 68$ & $873 \pm 21$ & $846 \pm 20$ \\
\hline $\begin{array}{l}\alpha \text {-tubulin and DRP } \\
\text { (inter- } \alpha \beta \text {-tubulin subunit) }\end{array}$ & $108 \pm 47$ & $81 \pm 31$ & - \\
\hline
\end{tabular}

*Interface areas were determined by a single buried surface, and averaged among each non-crystallographic unit in the structure.

drives the destabilization of the TOG square state and promotes the concerted unfurling of the TOG- $\alpha \beta$-tubulins to polymerize into a new protofilament.

\section{The polarized unfurling MT polymerase cycle}

We envision the polarized unfurling model as follow: 1) Upon recruiting four soluble $\alpha \beta$-tubulins, dimeric TOG1-TOG2 arrays in proteins such Alp14 or Stu2, organize into compact TOG square assemblies in solution (Figure 8A). These assemblies place $\alpha \beta$-tubulins in close proximity with each other in a near head-to-tail polarized while inhibiting spontaneous polymerization. This 'ready-topolymerize' orientation is due to the asymmetry in each TOG domain: $\alpha \beta$-tubulin interface and the unique head-to-tail assembly formed by two TOG1-TOG2 array subunits formed in the TOG square (Figure 2). 2) The $\alpha \beta$-tubulin-loaded TOG square assemblies diffuse along the MT lattice, mediated by an interaction of the SK-rich regions immediately C-terminal to TOG2, with acidic tubulin C-termini exposed on MT surfaces (Figure 8A). Proximity of the SK-rich region to the TOG array is essential for MT polymerase activity, and increasing its polypeptide distance causes MT polymerase defects (Geyer et alı, 2018; Al-Bassam et alı, 2012; Brouhard et alı, 2008). 3) When the $\alpha \beta$-tubulin-loaded TOG squares reach the $\beta$-tubulins exposed at MT plus-end protofilament tips, $\alpha$-tubulin of the TOG1- or TOG2-bound $\alpha \beta$-tubulin may polymerize with $\beta$-tubulin exposed at the MT plus-end tip (Figure 8B-l; Figure 2B). Although TOG squares may diffuse to MT minus-ends, docking onto $\alpha$ tubulin at MT minus-ends is highly sterically inhibited, precluding the possibility of $\alpha \beta$-tubulin TOG square docking onto MT minus-ends. The $\alpha \beta$-tubulin docking of either TOG1 or TOG2 onto $\beta$-tubulin at MT plus-ends is likely to be random. However, two features of TOG domains favor TOG1- $\alpha \beta$ tubulin over TOG $2 \alpha \beta$-tubulin in docking onto MT plus-ends: A) TOG1 is more likely to be fully occupied by $\alpha \beta$-tubulin due to its slow exchange and high affinity compared to the rapid exchange of $\alpha \beta$-tubulin onto TOG2 (Figure 1). B) Steric overlap with MT protofilaments develops only if the TOG square docks via TOG1- $\alpha \beta$-tubulin but not if TOG2- $\alpha \beta$-tubulin docks (Figure 7; Figure 8A-I and BI; Figure 7-figure supplement 1A). Thus, TOG squares docking to protofilament ends via TOG1-

Table 5. Intra and inter dimer curvature angles $\left(^{\circ}\right)$ of $\alpha \beta$-Tubulins observed in structures.

\begin{tabular}{|c|c|c|c|}
\hline & PDB ID & Intra dimer $(\alpha 1 \beta 1)$ angle $\left({ }^{\circ}\right)$ & Inter dimer $\left(\beta 1 \alpha_{2}\right)$ angle $\left({ }^{\circ}\right)$ \\
\hline Stathmin:RB3 complex with GTP & 3RYH & 9.2 & 10.3 \\
\hline Stathmin:RB3 complex with GDP & $1 \mathrm{SAO}$ & 13.0 & 13.5 \\
\hline$\alpha \beta$-tubulin:TOG1 complex with GTP & 4FFB & 13 & - \\
\hline$\alpha \beta$-Tubulin:TOG2 complex with GTP & $4 \cup 3 \mathrm{~J}$ & 13 & - \\
\hline$\alpha \beta$-Tubulin:DRP complex with GDP & $4 \mathrm{DRX}$ & 11.0 & - \\
\hline Sk-Alp14-monomer: $\alpha \beta$-Tubulin:DRP wheel with GDP & Current & 11.3 & - \\
\hline Sk-Alp14-monomer: $\alpha \beta$-Tubulin:DRP- $\Delta N$ with GDP & Current & 11.2 & 16.4 \\
\hline
\end{tabular}

${ }^{*}$ Curvature of $\alpha \beta$-tubulin interface were determined as previously described by Rice and Brouhard 2015.

DOI: https://doi.org/10.7554/eLife.38922.021 
A

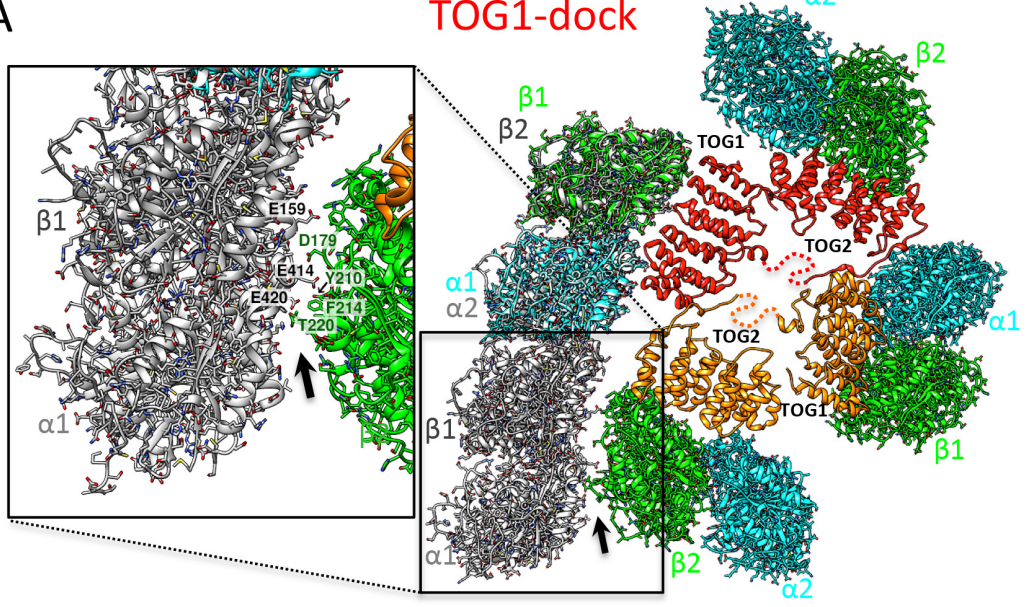

B

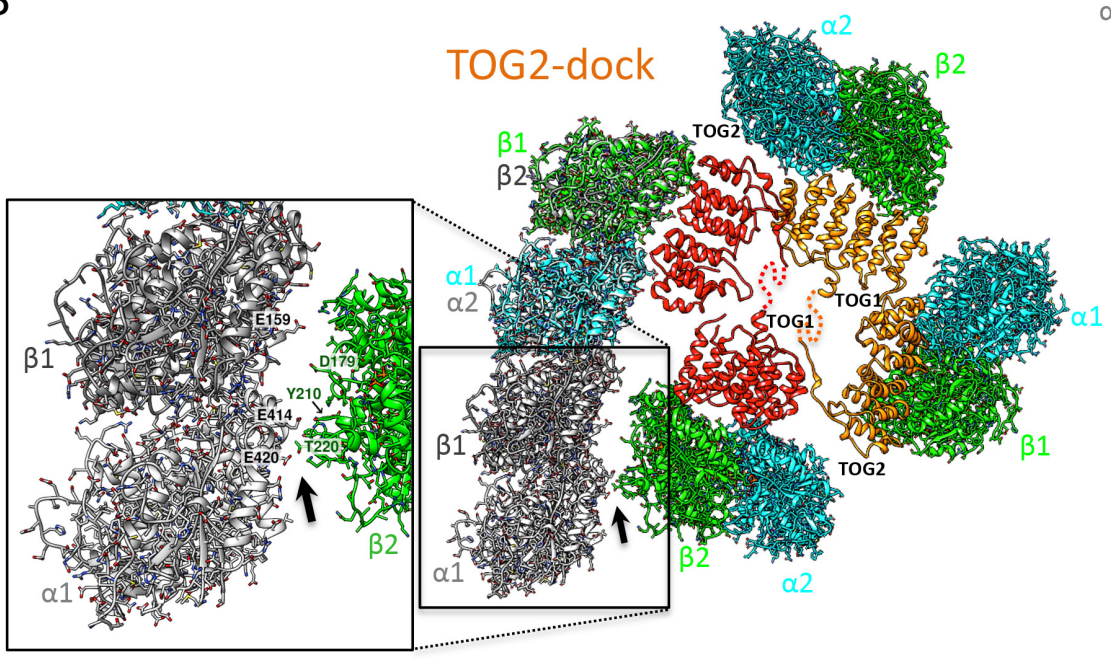

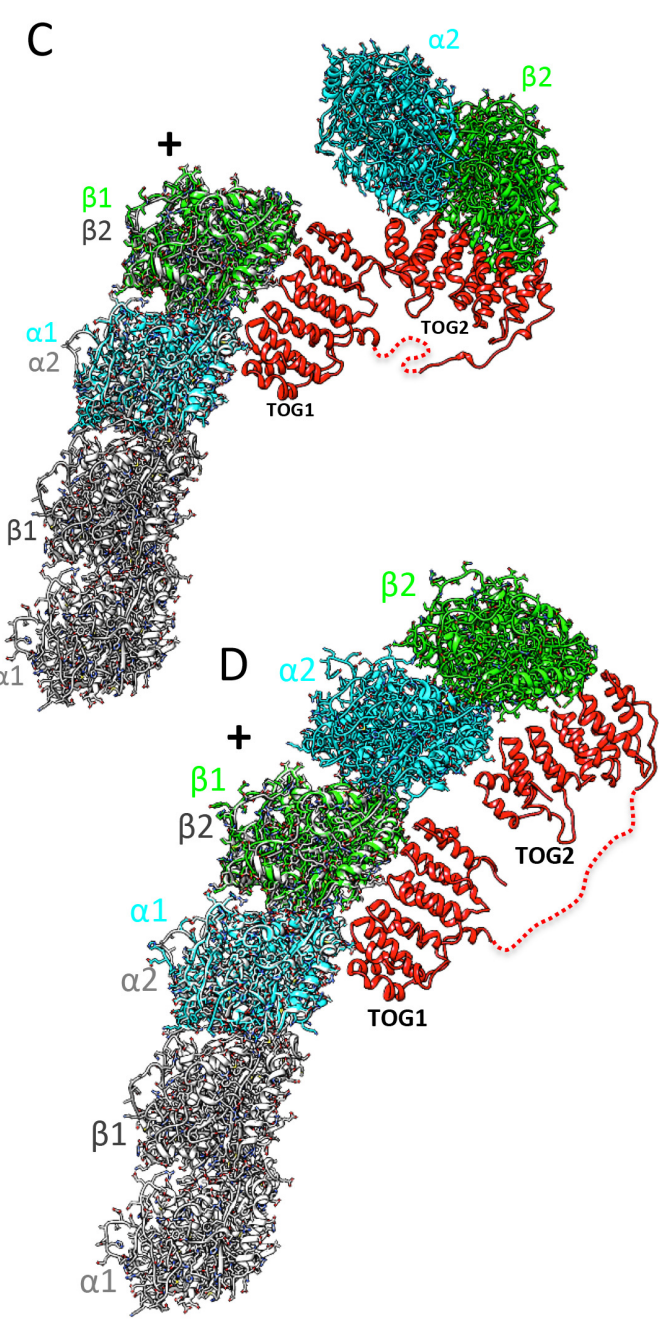

Figure 7. Docking of atomic structures onto protofilament ends reveals the molecular details of unfurling. (A) Right, atomic model for four $\alpha \beta$-tubulinbound TOG square X-ray structures (Figure 2) docked using $\alpha \beta$-tubulin bound to TOG1 at the terminal $\alpha \beta$-tubulin in a curved protofilament (PDB ID: $3 R Y H)$. Left, magnified view of the zone of steric contact between TOG2- $\alpha \beta$-tubulin in the second subunit and the penultimate $\alpha \beta$-tubulin of the protofilament below the polymerization site. (B) Right, atomic model for four $\alpha \beta$-tubulin-bound TOG square X-ray structure (Figure 2) docked using $\alpha \beta$ tubulin bound to TOG2 at the terminal $\alpha \beta$-tubulin in a curved protofilament (PDB ID: 3RYH). Left, magnified view of the zone shown in A between TOG1- $\alpha \beta$-tubulin in the second subunit and the penultimate $\alpha \beta$-tubulin of the protofilament. Details and overlay images are shown in Figure 7-figure supplement 1. (C) Docking the isolated TOG1-TOG2 two- $\alpha \beta$-tubulin assembly structure (extracted from the pseudo-dimer structure) onto the terminal $\alpha \beta$-tubulin of the curved protofilament. (D) Docking of the unfurled 1:2:1 unfurled assembly structure (Figure 5) to the curved protofilament revealing TOG1 to be positioned at the base of the new assembly while TOG2 is positioned at the outer end of the newly formed MT plus-end.

DOI: https://doi.org/10.7554/eLife.38922.023

The following figure supplement is available for figure 7 :

Figure supplement 1. All-atom docking models for structures superimposed onto curved protofilament plus-ends. DOI: https://doi.org/10.7554/eLife.38922.024

$\alpha \beta$-tubulin destabilizes the TOG square in contrast with TOG2: $\alpha \beta$-tubulin, which will not destabilize the TOG square at protofilament tips, leading to selection of TOG1 at the docking site (Figurer 8BII). 4) The MT plus-end-induced TOG square destabilization likely triggers TOG square disassembly into two corner-shaped TOG1-TOG2 subunits at MT plus-ends. 5) Corner-like half-square TOG1TOG2 subunit assemblies are then released which are likely unstable (Figure 8B-II), and interface 2 likely acts as a flexible hinge for TOG2 to freely rotate around TOG1, driven by Brownian motion. Reversible unfurling, or hinge rotation, promotes $\alpha \beta$-tubulin bound to TOG2 to polymerize 


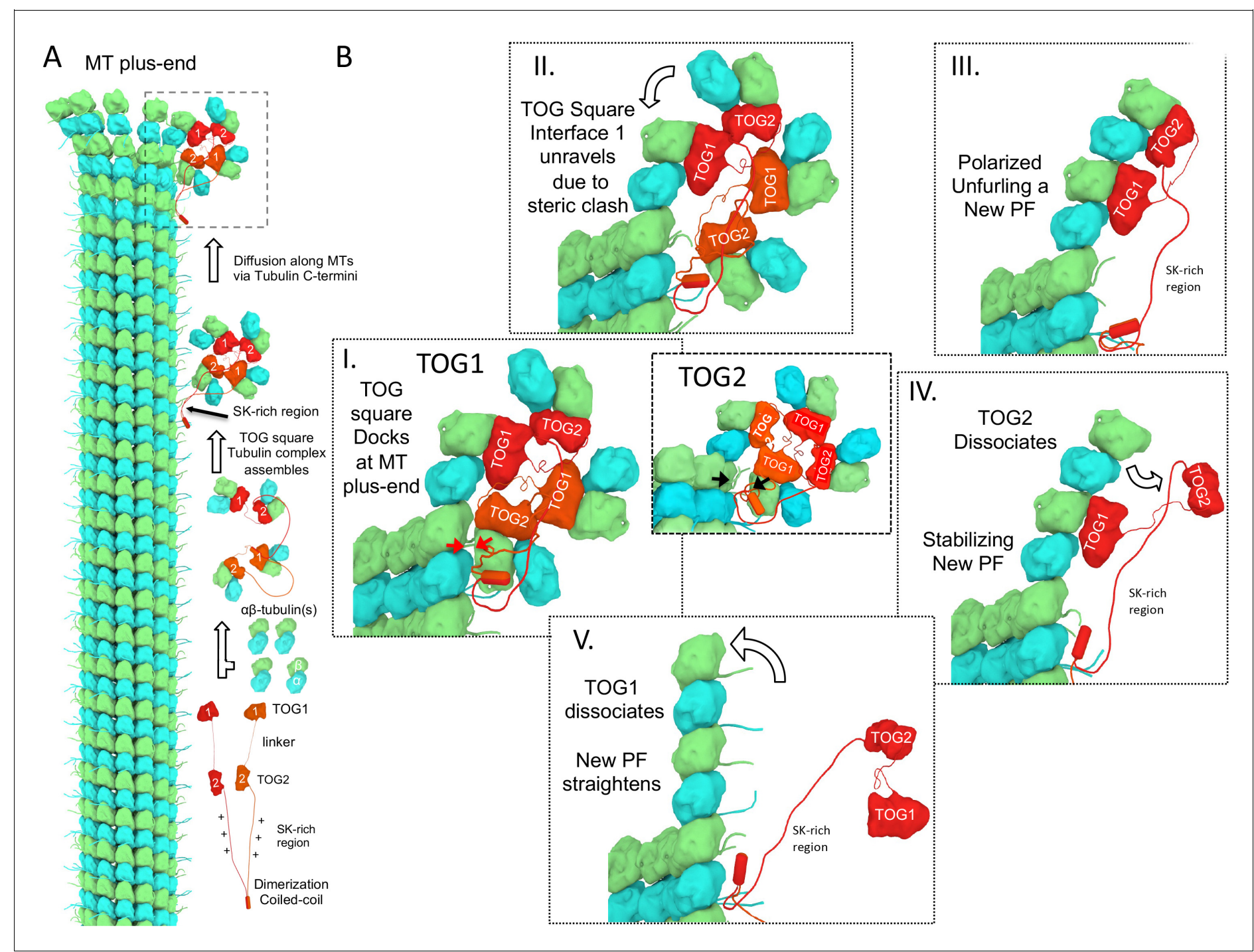

Figure 8. A polarized unfurling model for TOG arrays as MT polymerases. An animation for this model is shown in Video 1. (A) Assembly of yeast MT polymerase dimeric TOG1-TOG2 subunits with four $\alpha \beta$-tubulins into an $\alpha \beta$-tubulin:TOG square. TOG squares diffuse along MT lattices modulated by tubulin C-termini interacting with SK-rich regions. (B) I. TOG square assemblies orient $\alpha \beta$-tubulins in wheel-shaped assemblies at MT plus-ends. II. These assemblies are destabilized upon TOG1- $\alpha$-tubulin polymerizing onto the exposed $\beta$-tubulin at MT plus-ends, releasing TOG1-TOG2 subunits in corner conformations. III. The release of TOG2: $\alpha \beta$-tubulin allows free rotation around TOG1, driving two $\alpha \beta$-tubulins to polymerize. IV. TOG2 dissociates from the newly polymerized $\alpha \beta$-tubulin stabilizing protofilament at the plus-end while TOG1 anchors this $\alpha \beta$-tubulin onto the MT plus-end. V. Straightening of this new protofilament leads to the dissociation of TOG1. The rebinding of TOG1-TOG2 subunits to $\alpha \beta$-tubulins reforms the TOG square assembly and restarts the MT polymerase cycle. Atomic views for states I, II, and III are shown in Figure 7.

catalytically onto the plus-end of the TOG1-bound $\alpha \beta$-tubulin (Figure 8B-III). The TOG1- and TOG2-bound $\alpha \beta$-tubulins polymerize in a single concerted unfurling event as seen in the polymerized TOG1-TOG2: $\alpha \beta$-tubulin X-ray structure (Figure $5 E, F$ ). This event effectively 'unfurls' a single curved protofilament from two $\alpha \beta$-tubulins pre-oriented onto an $\alpha \beta$-tubulin-loaded TOG1-TOG2 corner-like intermediate. No energy expenditure is required during unfurling, as reversible Brownian motion likely drives the unfurling activity. However, formation of the polymerized assembly intermediate is captured by the $\alpha \beta$-tubulin- $\alpha \beta$-tubulin inter-dimer polymerization interfaces, which become locked by the inter-dimer interface conformational change, as seen in in the polymerized state structure (Figure $6 A, B)$. The $\alpha \beta$-tubulin inter-dimer interfaces (1650 $\AA^{2}$ surface area in a single interface) may compete with TOG square reformation (1930 $\AA^{2}$ in total for a TOG square). 6) A gradient in the 


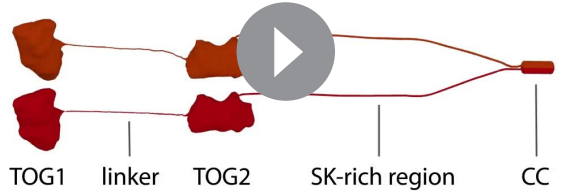

Video 1. Animation of the mechanism of polarized unfurling for multiple TOG domains in a yeast MT polymerase. This animation describes the 'polarized unfurling' mechanism for multiple TOG domains in promoting MT polymerization. Briefly, Yeast MT polymerases are dimers with each subunit including TOG1 and TOG2 domains separated by a linker and followed by unstructured SK-rich and coiled-coil domains. Each MT polymerase binds four $\alpha \beta$-tubulins forming TOG square assemblies, as shown in the ribbon diagram (Figure 2). TOG2 exchanges $\alpha \beta$ tubulin due to its rapid exchange rate, while TOG square assemblies diffuse along MT lattices loaded with $\alpha \beta$-tubulins at MT plus-ends, as visualized in Figure 8. Docking of TOG square assemblies via TOG2 $\alpha \beta$-tubulin does not destabilize the TOG square as described in Figure 7. The polymerization of $\alpha \beta$ tubulin-TOG1 destabilizes TOG square complexes at interface 1 due to steric contact as described in Figure 7. The destabilization of the TOG square soluble $\alpha \beta$-tubulins into one curved protofilament at the MT plus-end, as visualized in Figure 5. The newly formed protofilament is further stabilized by the rapid dissociation of TOG 2 from the outermost $\alpha \beta$-tubulin, and forms corners to enhance direct $\alpha \beta$-tubulin polymerization at the MT plus-end. Other MT polymerase molecules promote delivery of $\alpha \beta$-tubulin dimers via the 'polarized unfurling' mechanism at polymerizing MT plus-ends. Individual steps for this model are shown in Figure 8.

DOI: https://doi.org/10.7554/eLife.38922.026 releases TOG2, promoting polarized unfurling of two

$\alpha \beta$-tubulin exchange rates between TOG1 and TOG2 likely leads TOG2 to dissociate from its $\alpha \beta$-tubulin rapidly before TOG1 dissociates from its $\alpha \beta$-tubulin (Figure 1; Figure $8 \mathrm{~A}-\mathrm{VI}$ ). The unfurled TOG1-TOG2 $\alpha \beta$-tubulin polymerized structure positions this affinity gradient spatially across lower and upper positions of the polymerized complex with respect to the MT plus-end (Figure 6). A tightly bound $\alpha \beta$-tubulin on TOG1 likely anchors the TOG array onto the MT plusend while the rapidly exchanging $\alpha \beta$-tubulin promotes TOG2 release- an intermediate that promotes accelerated MT polymerase (Figure 8B IV). 10) Protofilament straightening during MT plus-end closure likely induces TOG1 dissociation from the lower $\alpha \beta$-tubulin of the newly polymerized protofilament as suggested previously by the 'catch and release' model (Ayaz et al., 2012). The unbound TOG1-TOG2 arrays are finally released from the newly formed protofilament to reload with soluble $\alpha \beta$-tubulin from the cytoplasm. TOG arrays may then reform the TOG square assembly upon recruiting $\alpha \beta$-tubulins and restart the cycle, while the SK-rich region maintains contact with the polymerizing MT plus-end (Figure 8B-V) (Video 1).

\section{Implications of 'polarized unfurling'}

The polarized unfurling model suggests that the MT polymerase activity of TOG arrays is due to three features: 1) pre-organization of $\alpha \beta$-tubulins onto the TOG square. promoted by interfaces 1 and 2. 2) MT plus-end-induced unfurling of TOG square via TOG1- $\alpha \beta$-tubulin induced destabilization (Figure 7). 3) Reversible unfurling driven by Brownian motion and the polymerization capacity of $\alpha \beta$-tubulins being recruited TOG1-TOG2 squares disassemble. The propensity of TOG1TOG2 subunit head-to-tail self-assembly is strongly enhanced by C-terminal coiled-coildimerization or the presence of TOG3-TOG4 in metazoan TOG arrays such as XMAP215/ch-TOG proteins. The positive charge of the SK-rich region is essential to associate with the MT surface. The polarized unfurling model suggests that TOG1 and TOG2 serve specific roles in MT polymerase activity, TOG1 anchors the array and destabilizes the TOG square organization onto the MT plus-end, while TOG2 drives $\alpha \beta$-tubulin polymerization.

\section{Comparison to other MT polymerase models}

Two other models were suggested for the functions of TOG arrays as MT polymerases. In these models, TOG1 and TOG2 domains were suggested to exhibit random or reversed orientations in the process of polymerizing $\alpha \beta$-tubulin, compared to the polarized unfurling model (Ayaz et al., 2014; Fox et al., 2014). Most recently, Geyer et al. (2018) studied the roles of TOG1 and TOG2 in MT polymerase activity by generating All-TOG1 or All-TOG2 chimeras of the budding yeast Stu2 MT polymerase. This study concluded that any two TOG domains, regardless of identity, are required in 
a TOG array for MT polymerase activity. This observation agrees with the importance of two adjacent TOG domains being critical for forming a two $\alpha \beta$-tubulin polymerized complex. However, the study concluded that no higher order of organization, such as the TOG square observed here, was occurring since TOG domain identity did not influence MT polymerase activity. However, our TOG square assembly structure indicates that the critical 12-residue linker adjacent to TOG2 still remains in these All-TOG1 or All-TOG2 Stu2 constructs. Thus, our studies demonstrate that the chimeric constructs used in that study (Geyer et al., 2018) still retained a substantial TOG square assembly capacity, despite exchanging TOG1 for TOG2 domain sequences. These chimeric Stu2 proteins were not structurally characterized in that study, and thus their self-assembly properties using their modified TOG arrays remain unknown (Geyer et al., 2018).

Our cysteine mutagenesis, crosslinking/mass spectrometry, and negative stain EM studies indicate wt-Alp14-dimer forms TOG squares upon binding $\alpha \beta$-tubulin in solution (Figures 3-4). Biochemical and negative stain-EM suggest that interfaces 1 and two organize TOG square assemblies, and that their inactivation in the INT1, INT2, and INT1 +2 mutants results in specific loss of either interface 1 or interface 2 producing either single corner-like subunits bound to two orthogonally oriented $\alpha \beta$-tubulins, two spontaneously polymerized $\alpha \beta$-tubulins bound TOG1-TOG2 arrays (INT1 and INT2), or disorganized arrays composed of multiple TOG- $\alpha \beta$-tubulins (INT1 +2); however these defects did not influence the ability of TOG arrays to bind $\alpha \beta$-tubulins. Our data support that interfaces 1 and 2 pre-orient $\alpha \beta$-tubulins and suggest that spontaneous polymerization may occur if interface 1 or interface 2 become destabilized, supporting our model (Figures 4-5). The polarized unfurling model explains a well-documented observation that could not be previously rationalized. Fusion of protein masses such as GFP protein, but not short tags, onto the TOG1 N-terminus severely inactivates MT polymerases, exclusively activating their MT depolymerase activity without affecting $\alpha \beta$-tubulin binding (Lechner et al., 2012) and references herein). Our model shows that N-terminal fusions on TOG arrays strongly interfere with formation of a TOG square by forming blocks that sterically hinder interface 2 formation.

\section{Comparison to other MT regulatory proteins with TOG arrays}

Other conserved classes of MT regulatory TOG-like array proteins, such as CLASP and Crescerin/ CHE-12, may form similar TOG square-like particles and modulate MT dynamics in related mechanisms. For instance, the $S$. pombe CLASP: $\alpha \beta$-tubulin complexes form wheel-like particles with similar dimensions and promote local MT rescue (Al-Bassam and Chang, 2011; Al-Bassam et al., 2010; Das et al., 2015). However, the high-resolution organization of these different TOG arrays remains to be determined at high resolution.

\section{Materials and methods}

Key resources table

\begin{tabular}{|c|c|c|c|c|}
\hline $\begin{array}{l}\text { Reagent type } \\
\text { (species) } \\
\text { or resource } \\
\end{array}$ & Designation & Source & Identifier & $\begin{array}{l}\text { Additional } \\
\text { information }\end{array}$ \\
\hline $\begin{array}{l}\text { Chemical } \\
\text { compound, } \\
\text { drug }\end{array}$ & $\begin{array}{l}\text { Darpin D1 } \\
\text { (Synthetic DNA) }\end{array}$ & Invitrogen & $\mathrm{N} / \mathrm{A}$ & \\
\hline $\begin{array}{l}\text { Chemical } \\
\text { compound, } \\
\text { drug }\end{array}$ & GTP & Sigma & G-8877 & \\
\hline $\begin{array}{l}\text { Chemical } \\
\text { compound, } \\
\text { drug }\end{array}$ & GDP & Sigma & G7127 & \\
\hline $\begin{array}{l}\text { Chemical } \\
\text { compound, } \\
\text { drug }\end{array}$ & $\begin{array}{l}\text { Crystallization } \\
\text { plates }\end{array}$ & $\begin{array}{l}\text { TTP } \\
\text { Labtech }\end{array}$ & 4150-05600 & \\
\hline $\begin{array}{l}\text { Chemical } \\
\text { compound, } \\
\text { drug }\end{array}$ & $\begin{array}{l}\text { Crystallization } \\
\text { sparse matrix } \\
\text { screens }\end{array}$ & Qiagen & N/A & \\
\hline
\end{tabular}

Continued on next page 
Continued

\begin{tabular}{|c|c|c|c|c|}
\hline $\begin{array}{l}\text { Reagent type } \\
\text { (species) } \\
\text { or resource }\end{array}$ & Designation & Source & Identifier & $\begin{array}{l}\text { Additional } \\
\text { information }\end{array}$ \\
\hline $\begin{array}{l}\text { Chemical } \\
\text { compound, } \\
\text { drug }\end{array}$ & PEG-8000 & Sigma & 1546605 & \\
\hline $\begin{array}{l}\text { Chemical } \\
\text { compound, } \\
\text { drug }\end{array}$ & PEG-2000 & Sigma & 8.21037 & \\
\hline $\begin{array}{l}\text { Chemical } \\
\text { compound, } \\
\text { drug }\end{array}$ & $\begin{array}{l}\text { Copper(II) } \\
\text { sulfate }\end{array}$ & Sigma & C1297 & \\
\hline $\begin{array}{l}\text { Chemical } \\
\text { compound, } \\
\text { drug }\end{array}$ & $\begin{array}{l}\text { 1, 10- } \\
\text { phenanthroline }\end{array}$ & Sigma & 131377 & \\
\hline $\begin{array}{l}\text { Chemical } \\
\text { compound, } \\
\text { drug }\end{array}$ & Trypsin & Sigma & T6567 & \\
\hline $\begin{array}{l}\text { Chemical } \\
\text { compound, } \\
\text { drug }\end{array}$ & Chymotrypsin & Sigma & C6423 & \\
\hline $\begin{array}{l}\text { Chemical } \\
\text { compound, } \\
\text { drug }\end{array}$ & lodoacetamide & Sigma & 16125 & \\
\hline $\begin{array}{l}\text { Chemical } \\
\text { compound, } \\
\text { drug }\end{array}$ & 4-Vinylpyridine & Sigma & V3204 & \\
\hline Other & $\begin{array}{l}\text { 2:4:4 sk- } \\
\text { Alp14-550: } \alpha \beta- \\
\text { Tubulin:DRP }\end{array}$ & $\begin{array}{l}\text { Protein } \\
\text { Data Bank }\end{array}$ & PDB: \#6MZF & $\begin{array}{l}\text { Deposited Data } \\
\text { (Atomic } \\
\text { coordinates) }\end{array}$ \\
\hline Other & $\begin{array}{l}\text { 2:4:4 } \\
\text { sk-Alp14-550- } \\
\text { SL: } \alpha \beta-T u b u l i n: \\
\text { DRP }\end{array}$ & $\begin{array}{l}\text { Protein } \\
\text { Data Bank }\end{array}$ & PDB: \#6MZE & $\begin{array}{l}\text { Deposited } \\
\text { Data (Atomic } \\
\text { coordinates) }\end{array}$ \\
\hline Other & $\begin{array}{l}\text { 1:2:1 } \\
\text { sk-Alp14-550: } \\
\alpha \beta-T u b u l i n: D R P \Delta N\end{array}$ & $\begin{array}{l}\text { Protein } \\
\text { Data Bank }\end{array}$ & PDB: \#6MZG & $\begin{array}{l}\text { Deposited } \\
\text { Data (Atomic } \\
\text { coordinates) }\end{array}$ \\
\hline Other & $\begin{array}{l}\text { Saccharomyces } \\
\text { cerevisiae Stu2p }\end{array}$ & $\begin{array}{l}\text { UniprotKB/ } \\
\text { Swiss-Prot }\end{array}$ & P46675 & $\begin{array}{l}\text { Protein } \\
\text { sequence }\end{array}$ \\
\hline Other & $\begin{array}{l}\text { Saccharomyces kluyveri } \\
\text { Stu2p or Alp14p }\end{array}$ & $\begin{array}{l}\text { Lachancea kluyveri } \\
\text { NRRL } \\
\text { Y-12651 } \\
\text { chromosome }\end{array}$ & SKLU-Cont10078 & Protein sequence \\
\hline Other & $\begin{array}{l}\text { Schizos } \\
\text { accharomyces } \\
\text { pombe Alp 14p }\end{array}$ & $\begin{array}{l}\text { UniprotKB/ } \\
\text { Swiss-Prot }\end{array}$ & 094534 & Protein sequence \\
\hline Other & $\begin{array}{l}\text { Chaetomium } \\
\text { thermophilum } \\
\text { Stu2 }\end{array}$ & $\begin{array}{l}\text { UniprotKB/ } \\
\text { Swiss-Prot }\end{array}$ & G0S3A7 & Protein sequence \\
\hline Other & $\begin{array}{l}\text { SoluBL21 } \\
\text { bacterial } \\
\text { expression } \\
\text { system }\end{array}$ & AmsBio & C700200 & $\begin{array}{l}\text { Model } \\
\text { system } \\
\text { (expression } \\
\text { system) }\end{array}$ \\
\hline $\begin{array}{l}\text { Recombinant } \\
\text { DNA reagent }\end{array}$ & $\begin{array}{l}\text { pLIC_V2-Sc } \\
\text { Stu2p- } H_{6}\end{array}$ & $\begin{array}{l}\text { Current } \\
\text { study }\end{array}$ & $N / A$ & $\begin{array}{l}\text { Recombinant } \\
\text { DNA constructs } \\
\text { Expressed } \\
\text { in bacterial } \\
\text { strains }\end{array}$ \\
\hline $\begin{array}{l}\text { Recombinant } \\
\text { DNA reagent }\end{array}$ & $\begin{array}{l}\text { pLIC_V2-Sk } \\
\text { Stu2p- } H_{6}\end{array}$ & $\begin{array}{l}\text { Current } \\
\text { study }\end{array}$ & $N / A$ & \\
\hline
\end{tabular}

\section{Continued on next page}


Continued

\begin{tabular}{|c|c|c|c|c|}
\hline $\begin{array}{l}\text { Reagent type } \\
\text { (species) } \\
\text { or resource }\end{array}$ & Designation & Source & Identifier & $\begin{array}{l}\text { Additional } \\
\text { information }\end{array}$ \\
\hline $\begin{array}{l}\text { Recombinant } \\
\text { DNA reagent }\end{array}$ & $\begin{array}{l}\text { pLIC_V2-Sc } \\
\text { Stu2-550-H } \\
\text { (TOG1-TOG2 } \\
\text { monomer) }\end{array}$ & $\begin{array}{l}\text { Al-Bassam } \\
\text { et al., } 2006\end{array}$ & & \\
\hline $\begin{array}{l}\text { Recombinant } \\
\text { DNA reagent }\end{array}$ & $\begin{array}{l}\text { pLIC_V2-KL-Stu2 } \\
\text {-monomer-H } \\
\text { (residues } 1-560 \text { ) }\end{array}$ & $\begin{array}{l}\text { Current } \\
\text { study }\end{array}$ & NA & \\
\hline $\begin{array}{l}\text { Recombinant } \\
\text { DNA reagent }\end{array}$ & $\begin{array}{l}\text { pLIC_V2-CT } \\
\text { Stu2-mon } \\
\text { omer-H } \\
\text { (residues 1-550) }\end{array}$ & $\begin{array}{l}\text { Current } \\
\text { study }\end{array}$ & NA & \\
\hline $\begin{array}{l}\text { Recombinant } \\
\text { DNA reagent }\end{array}$ & $\begin{array}{l}\text { PLIC_V2-SK } \\
\text { Alp14-monomer- } \\
\mathrm{H}_{6} \text { (residues 1-550) }\end{array}$ & $\begin{array}{l}\text { Current } \\
\text { study }\end{array}$ & N/A & \\
\hline $\begin{array}{l}\text { Recombinant } \\
\text { DNA reagent }\end{array}$ & $\begin{array}{l}\text { PLIC_V2-Sk } \\
\text { Alp14-monomer } \\
\text {-SL-Ho } \\
\text { (residues 1-550; } \\
\text { linker } \\
\text { residues replaced } \\
\text { KL sequence; } \\
\text { see Materials } \\
\text { and methods) }\end{array}$ & $\begin{array}{l}\text { Current } \\
\text { study }\end{array}$ & N/A & \\
\hline $\begin{array}{l}\text { Recombinant } \\
\text { DNA reagent }\end{array}$ & $\begin{array}{l}\text { pLIC_V2- } \\
\text { sk-wt-Alp14- } \\
\text { dimer-H6 } \\
\text { (residues 1-724) }\end{array}$ & $\begin{array}{l}\text { Current } \\
\text { study }\end{array}$ & N/A & \\
\hline $\begin{array}{l}\text { Recombinant } \\
\text { DNA reagent }\end{array}$ & $\begin{array}{l}\text { pLIC_V2 } \\
\text {-sk-Alp14- } \\
\text { dimer- } \mathrm{H}_{6}\end{array}$ & $\begin{array}{l}\text { Current } \\
\text { study }\end{array}$ & N/A & \\
\hline $\begin{array}{l}\text { Recombinant } \\
\text { DNA reagent }\end{array}$ & $\begin{array}{l}\mathrm{S} 180 \mathrm{C} \\
\text { and L304C } \\
\text { (residues } \\
1-724 \text { ) }\end{array}$ & & & \\
\hline $\begin{array}{l}\text { Recombinant } \\
\text { DNA reagent }\end{array}$ & $\begin{array}{l}\text { pLIC_V2- } \\
\text { sk-Alp14- } \\
\text { dimer- } H_{6}\end{array}$ & Current study & N/A & \\
\hline $\begin{array}{l}\text { Recombinant } \\
\text { DNA reagent }\end{array}$ & $\begin{array}{l}\text { S41C and } \\
\text { E518C } \\
\text { (residues } \\
1-724 \text { ) }\end{array}$ & & & \\
\hline $\begin{array}{l}\text { Recombinant } \\
\text { DNA reagent }\end{array}$ & $\begin{array}{l}\text { pLIC_V2-wt } \\
\text {-Alp14-dimer- } \\
\mathrm{H}_{6} \text { (residues } \\
1-690 \text { ) }\end{array}$ & Current study & N/A & \\
\hline $\begin{array}{l}\text { Recombinant } \\
\text { DNA reagent }\end{array}$ & $\begin{array}{l}\text { pLIC_V2-TOG1M } \\
\text { - } \text { H }_{6} \text { (residues } \\
\text { 1-690: Y23A } \\
\text { and R23A) }\end{array}$ & Current study & N/A & \\
\hline $\begin{array}{l}\text { Recombinant } \\
\text { DNA reagent }\end{array}$ & $\begin{array}{l}\text { pLIC_V2-TOG2M } \\
-\mathrm{H}_{6} \text { (residues } \\
\text { 1-690: Y300A } \\
\text { and K381A) }\end{array}$ & Current study & N/A & \\
\hline
\end{tabular}

\section{Continued on next page}


Continued

\begin{tabular}{|c|c|c|c|c|}
\hline $\begin{array}{l}\text { Reagent type } \\
\text { (species) } \\
\text { or resource }\end{array}$ & Designation & Source & Identifier & $\begin{array}{l}\text { Additional } \\
\text { information }\end{array}$ \\
\hline $\begin{array}{l}\text { Recombinant } \\
\text { DNA reagent }\end{array}$ & $\begin{array}{l}\text { pLIC_V2-INT1-H } \\
\text { (residues 1-690: } \\
\text { L206A, L208A, } \\
\text { F275R D276A, } \\
\text { L277A, V278A, } \\
\text { K320L, R359A) }\end{array}$ & Current study & $N / A$ & \\
\hline $\begin{array}{l}\text { Recombinant } \\
\text { DNA reagent }\end{array}$ & $\begin{array}{l}\text { pLIC_V2-INT2-H } \\
\text { (residues 1-690: } \\
\text { L39D, S40A, } \\
\text { D42A, L437D, } \\
\text { S440A, E478A } \\
\text { and R479A) }\end{array}$ & Current study & N/A & \\
\hline $\begin{array}{l}\text { Recombinant } \\
\text { DNA reagent }\end{array}$ & $\begin{array}{l}\text { pLIC_V2 } \\
\text {-INT1 + } 2 \mathrm{H}_{6} \\
\text { (L206A, L208A, } \\
\text { F275R D276A, } \\
\text { L277A, V278A, } \\
\text { K320L, R359A } \\
\text { L39D, S40A, } \\
\text { D42A, L437D, } \\
\text { S440A, E478A } \\
\text { and R479A) }\end{array}$ & Current study & $\mathrm{N} / \mathrm{A}$ & \\
\hline $\begin{array}{l}\text { Recombinant } \\
\text { DNA reagent }\end{array}$ & pET303-Ho-DRP & $\begin{array}{l}\text { Current } \\
\text { study }\end{array}$ & $\mathrm{N} / \mathrm{A}$ & \\
\hline $\begin{array}{l}\text { Recombinant } \\
\text { DNA reagent }\end{array}$ & $\mathrm{pLIC} \mathrm{V}_{2}-\mathrm{H}_{6}-\mathrm{DRP} \Delta \mathrm{N}$ & $\begin{array}{l}\text { Current } \\
\text { study }\end{array}$ & $\mathrm{N} / \mathrm{A}$ & \\
\hline Other & $\begin{array}{l}\alpha \beta \text {-tubulin } \\
\text { purified } \\
\text { from porcine } \\
\text { brains }\end{array}$ & $\begin{array}{l}\text { Current } \\
\text { study }\end{array}$ & $\mathrm{N} / \mathrm{A}$ & $\begin{array}{l}\text { Native protein } \\
\text { purification }\end{array}$ \\
\hline Other & $\begin{array}{l}\alpha \beta \text {-tubulin } \\
\text { purified from } \\
\text { porcine brains }\end{array}$ & Castoldi and Popov, 2003 & & \\
\hline $\begin{array}{l}\text { Software, } \\
\text { algorithm }\end{array}$ & ASTRA V6.0 & $\begin{array}{l}\text { Wyatt } \\
\text { Technology }\end{array}$ & $\begin{array}{l}\text { http://www.wyatt. } \\
\text { com/products/ } \\
\text { software/astra.html }\end{array}$ & \\
\hline $\begin{array}{l}\text { Software, } \\
\text { algorithm }\end{array}$ & NanoAnalyze & $\begin{array}{l}\text { TA } \\
\text { Instruments }\end{array}$ & $\begin{array}{l}\text { http://www.t } \\
\text { ainstruments.com/ }\end{array}$ & \\
\hline $\begin{array}{l}\text { Software, } \\
\text { algorithm }\end{array}$ & EMAN2 & & $\begin{array}{l}\text { http://blake.bcm } \\
\text {.edu/emanwiki/EMAN2 }\end{array}$ & \\
\hline $\begin{array}{l}\text { Software, } \\
\text { algorithm }\end{array}$ & iMOSFLM & Battye et al., 2011 & $\begin{array}{l}\text { http://www. } \\
\text { mrc-lmb.cam.ac. } \\
\text { uk/harry/imosflm/ver7 } \\
\text { 21/quickguide.html }\end{array}$ & \\
\hline $\begin{array}{l}\text { Software, } \\
\text { algorithm }\end{array}$ & PHASER & Terwilliger, 2000 & $\begin{array}{l}\text { http://www.p } \\
\text { haser.cimr.cam.ac. } \\
\text { uk/index.php/ }\end{array}$ & \\
\hline $\begin{array}{l}\text { Software, } \\
\text { algorithm }\end{array}$ & PHASER & McCoy, 2007 & $\begin{array}{l}\text { Phaser } \\
\text { _Crystallographic_ } \\
\text { Software }\end{array}$ & \\
\hline $\begin{array}{l}\text { Software, } \\
\text { algorithm }\end{array}$ & PyMol & Schrodinger, LLC & $\begin{array}{l}\text { http://www } \\
\text {.pymol.org/ }\end{array}$ & \\
\hline $\begin{array}{l}\text { Software, } \\
\text { algorithm }\end{array}$ & UCSF-Chimera & Pettersen et al., 2004 & $\begin{array}{l}\text { https://www.c } \\
\text { gl.ucsf.edu/chimera/ }\end{array}$ & \\
\hline $\begin{array}{l}\text { Software, } \\
\text { algorithm }\end{array}$ & DM from CCP4 suite & Cowtan and Main, 1996 & $\begin{array}{l}\text { http://ww } \\
\text { w.ccp4.ac.uk/html/ } \\
\text { dmmulti.html }\end{array}$ & \\
\hline
\end{tabular}

Continued on next page 
Continued

\begin{tabular}{|c|c|c|c|c|}
\hline $\begin{array}{l}\text { Reagent type } \\
\text { (species) } \\
\text { or resource } \\
\end{array}$ & Designation & Source & Identifier & $\begin{array}{l}\text { Additional } \\
\text { information }\end{array}$ \\
\hline $\begin{array}{l}\text { Software, } \\
\text { algorithm }\end{array}$ & PHENIX & Adams et al., 2010 & $\begin{array}{l}\text { https://w } \\
\text { ww.phenix-onlin } \\
\text { e.org }\end{array}$ & \\
\hline $\begin{array}{l}\text { Software, } \\
\text { algorithm }\end{array}$ & anisotropy server & Strong et al., 2006 & $\begin{array}{l}\text { https://servi } \\
\text { ces.mbi.ucla.edu/a } \\
\text { nisoscale/ }\end{array}$ & \\
\hline $\begin{array}{l}\text { Software, } \\
\text { algorithm }\end{array}$ & $\begin{array}{l}\text { Phyre protein } \\
\text { homology model }\end{array}$ & Kelley et al., 2015 & $\begin{array}{l}\text { www.sbg } \\
\text {.bio.ic.ac.uk/phyre2/ht } \\
\text { ml/page.cgi?id=index }\end{array}$ & \\
\hline $\begin{array}{l}\text { Software, } \\
\text { algorithm }\end{array}$ & Cr-yolo & Wagner et al., 2018 & $\begin{array}{l}\text { http://sphire. } \\
\text { mpg.de/wiki/ }\end{array}$ & \\
\hline $\begin{array}{l}\text { Software, } \\
\text { algorithm }\end{array}$ & Relion 2.2 & Kimanius et al., 2016 & $\begin{array}{l}\text { https://www } \\
\text { 2.mrc-Imb.cam.ac.uk/r } \\
\text { elion/index.php }\end{array}$ & \\
\hline $\begin{array}{l}\text { Software, } \\
\text { algorithm }\end{array}$ & Cryosparc & Punjani et al., 2017 & $\begin{array}{l}\text { https://c } \\
\text { ryosparc.com/ }\end{array}$ & \\
\hline $\begin{array}{l}\text { Software, } \\
\text { algorithm }\end{array}$ & MolProbity & Chen et al., 2012 & $\begin{array}{l}\text { http://molprob } \\
\text { ity.biochem.duke.edu }\end{array}$ & \\
\hline $\begin{array}{l}\text { Software, } \\
\text { algorithm }\end{array}$ & Coot & Emsley et al., 2010 & $\begin{array}{l}\text { http://www2 } \\
\text { mrc-Imb.cam.ac.u } \\
\text { k/personal/pe } \\
\text { msley/coot/ }\end{array}$ & \\
\hline $\begin{array}{l}\text { Software, } \\
\text { algorithm }\end{array}$ & $\begin{array}{l}\text { BLENDER } \\
\text { 3D-animation }\end{array}$ & $\begin{array}{l}\text { Blender } \\
\text { foundation }\end{array}$ & $\begin{array}{l}\text { https://ww } \\
\text { w.blender.org/ }\end{array}$ & \\
\hline
\end{tabular}

\section{Protein expression and purification of Alp14 and sk-Alp14 proteins}

The coding regions for MT polymerases from S. pombe Alp14p (accession: BAA84527.1), S. cerevisiae Stu2p (accession: CAA97574.1), Saccharomyces kluyveri Alp14 or Stu2p (coding region identified in accession: SKLU-Cont10078), and Chaetomium thermophilum Stu2p (accession: XP_006692509) were inserted into bacterial expression vectors with a C-terminal His-tag. wt-Alp14monomer (residues 1-510), wt-Alp14-dimer (residues 1-690), sk-Alp14-monomer (residues 1-550), sk-Alp14-dimer (residues 1-724), Sc-Stu2-dimer (residues 1-746), and Ct-Stu2-dimer (residues 1719) constructs were generated, including with or without the SK-rich and coiled-coil dimerization regions. TOG1M and TOG2M mutants were generated via point mutagenesis of $Y 23 \mathrm{~A}$ and R23A to inactivate TOG1 domains (TOG1M); and Y300A and K381A to inactivate TOG2 domains (TOG2M) (Al-Bassam et alo, 2012). The INT1 +2 mutant was generated via gene synthesis (Epoch Life Science) by introducing 15-residue mutations into the wt-Alp14-dimer sequence (L206A L208A F275R D276A L277A V278A K320L R359A L39D S40A D42A L437D S440A E478A R479A). The INT1 and INT2 mutants were generated by a PCR swapping strategy of INT1 +2 with wt-Alp14-dimer leading to INT1 with 8-residue mutations (L206A L208A F275R D276A L277A V278A K320L R359A) and INT2 with 7-residue mutations (L39D S40A D42A L437D S440A E478A R479A). Generally, constructs were transformed and expressed in BL21 bacterial strains using the T7 expression system, and were grown at $37^{\circ} \mathrm{C}$ and induced with $0.5 \mathrm{mM}$ isopropyl thio- $\beta$-glycoside at $18^{\circ} \mathrm{C}$ overnight. Cells were centrifuged and then lysed using a microfluidizer (Avastin). Extracts were clarified via centrifugation at $18,000 \times g$. Proteins were purified using Ni-IDA (Macherey-Nagel) and/or ion exchange using Hitrap-SP or Hitrap-Q chromatography followed by size exclusion chromatography using a Superdex 200 (30/1000) column (GE Healthcare). DRP was synthesized (Gene Art, Life Technologies), inserted into bacterial expression vectors with a C-terminal $6 \times \mathrm{His}$ tag, and expressed as described above. Proteins were purified using Ni-NTA (Macherey-Nagel) followed by Hitrap $Q$ ion exchange and followed by size exclusion chromatography as described above. Purified proteins were used immediately or frozen in liquid nitrogen for future use. 


\section{Biochemical analyses of Alp14: $\alpha$-tubulin complexes}

Soluble porcine $\alpha \beta$-tubulin $(10 \mu \mathrm{M}$ or $20 \mu \mathrm{M})$ purified using two GTP-polymerization cycles at high ionic strength as previously described (Castoldi and Popov, 2003) was mixed with $5 \mu \mathrm{M} S$. kluyveri (sk) or S. pombe wt-Alp14-monomer, wt-Alp14-dimer, TOG1M, TOG2M, INT1, INT2, or INT1 +2 mutant proteins and then diluted five-fold. To assess $\alpha \beta$-tubulin assembly, the protein mixtures were analyzed by mixing the proteins into $0.5 \mathrm{~mL}$ volumes and injecting them into a Superdex 200 (10/ 300) size exclusion chromatography (SEC) column equilibrated in $100 \mathrm{mM}$ or $200 \mathrm{mM} \mathrm{KCl}$ binding buffer $\left(50 \mathrm{mM}\right.$ HEPES [pH 7.0], $1 \mathrm{mM} \mathrm{MgCl}_{2}$, and $1 \mathrm{mM} \beta$-mercaptoethanol with $100 \mathrm{mM} \mathrm{KCl}$ or 200 $\mathrm{mM} \mathrm{KCl}$ ) using an AKTA purifier system (GE Healthcare). Elution fractions $(0.5 \mathrm{~mL})$ were collected and analyzed via sodium dodecyl sulfate polyacrylamide gel electrophoresis (Bio-Rad). The $\alpha \beta$-tubulin- and Alp14-containing bands were quantitated using densitometry to determine the amounts of bound and unbound $\alpha \beta$-tubulin in each SEC fraction. Molecular masses of wt-Alp14-monomer, wtAlp14-dimer, TOG1M, TOG2M, INT1, INT2, and INT1 +2 proteins, $\alpha \beta$-tubulin, and their complexes were measured using SEC-coupled multi-angle light scattering (SEC-MALS). Complexes were separated on Superdex 200 Increase (10/300) columns (GE Healthcare) while measuring UV absorbance (Agilent 1100-Series HPLC), light scattering (Wyatt Technology miniDAWN TREOS), and refractive index (Wyatt Technology Optilab T-rEX). Concentration-weighted molecular masses for each peak were calculated using ASTRA six software (Wyatt Technology).

Isothermal titration calorimetery (ITC) was performed using a Nano-ITC (TA Instruments) to determine DRP and DRP $\Delta N$ affinities for $\alpha \beta$-tubulin. Experiments were performed at $25^{\circ} \mathrm{C}$. Soluble $\alpha \beta$ tubulin, DRP, and DRP $\Delta \mathrm{N}$ were diluted in $50 \mathrm{mM}$ HEPES buffer, $\mathrm{pH} 7.3,100 \mathrm{mM} \mathrm{KCl}, 1 \mathrm{mM} \mathrm{MgCl}$, and $50 \mu \mathrm{M}$ GDP. The sample cell was filled with tubulin (20-40 $\mu \mathrm{M})$ for every experiment. 135-250 $\mu \mathrm{M}$ of DRP or DRP $\Delta \mathrm{N}$ solutions were injected in volumes of 2 or $5 \mu \mathrm{L}$ in a series of controlled doses into the sample cell. To determine TOG1 and TOG2 affinities for $\alpha \beta$-tubulin with DRP, proteins were diluted in $50 \mathrm{mM}$ HEPES buffer, $\mathrm{pH} 7.3,100$ or $200 \mathrm{mM} \mathrm{KCl}$, and $1 \mathrm{mM} \mathrm{MgCl}$. $100-250 \mu \mathrm{M}$ of TOG1 or TOG2 solutions were injected in volumes of 2 or $5 \mu \mathrm{L}$ in a series of controlled does into the sample cell containing 1:1 molar ratio of $\alpha \beta$-tubulin and DRP $(20-40 \mu \mathrm{M})$. The results were analyzed with NanoAnalyze software (TA Instruments). Thermodynamic parameters were calculated using the standard thermodynamic equation: $-R T \ln K_{a}=\Delta G=\Delta H-T \Delta S$, where $\Delta G, \Delta H$, and $\Delta S$ are the changes in free energy, enthalpy, and entropy of binding, respectively, $T$ is the absolute temperature, and $R$ is the gas constant $\left(1.98 \mathrm{cal} \mathrm{mol}^{-1} \mathrm{~K}^{-1}\right)$.

\section{Crystallization of sk-Alp14: $\alpha \beta$-tubulin:DRP or drpon complexes}

Complexes $(200 \mu \mathrm{M})$ were screened for crystallization using commercial sparse matrix (Qiagen) or homemade screens in 96-well format using a Mosquito robot (TTP Labtech) via the hanging drop method. Cube-shaped crystals (5 $\mu \mathrm{m}$ on each edge) formed for sk-Alp14-monomer: $\alpha \beta$-tubulin:DRP complexes and grew over 4-7 days in $50 \mathrm{mM}$ PIPES, $100 \mathrm{mM} \mathrm{MgCl}$ [pH 7.0], and 10-15\% PEG8000. Larger crystals were grown using micro-seeding (Figure 2-figure supplement 2A). To obtain improved X-ray diffraction (see below), we used an sk-Alp14-monomer construct in which non-conserved 256-297 residue linkers were replaced by the shorter linker (including the residue sequence AVPAQSDNNSTLQTDKDGDTLMGN-) from the $K$. lactis ortholog sequence (termed sk-Alp14-monomer-SL). Crystals were transferred to $50 \mathrm{mM}$ PIPES, $100 \mathrm{mM} \mathrm{MgCl} 2$ [pH 7.0], $15 \%$ PEG-8000, and $25 \%$ glycerol for cryo-protection and flash frozen in liquid nitrogen.

Rectangular crystals of sk-Alp14-monomer: $\alpha \beta$-tubulin:DRP $\Delta \mathrm{N}$ complexes formed in 7-10 days under the same conditions described for cube-shaped TOG1-TOG2: $\alpha \beta$-tubulin:DRP crystals. These rectangular crystals exclusively formed using DRP $\Delta N$ (did not form with DRP) and were obtained using a variety of constructs of monomeric as well as dimeric sk-Alp14-monomer (Table 3). Rectangular sk-Alp14-monomer: $\alpha \beta$-tubulin:DRP $\Delta \mathrm{N}$ crystals were treated for cryo-protection and flash frozen as described above.

\section{X-ray diffraction and structure determination of sk-Alp14: $\alpha \beta$-tubulin assemblies}

More than 100 sk-Alp14-monomer: $\alpha \beta$-tubulin:DRP crystals were screened for X-ray diffraction at the Argonne National Laboratory at the Advanced Photon Source microfocus 24-ID-C beamline. Anisotropic X-ray diffraction data were collected for the best cube-shaped crystals in the $P 2_{1}$ space group 
to $4.4 \AA$ resolution in the best dimension, with unit cell dimensions $a=219 \AA, b=108 \AA$, and $c=283 \AA$ (Figure 2-figure supplement 1). The sk-Alp14-monomer-SL: $\alpha \beta$-tubulin:DRP crystals showed improved diffraction and decreased anisotropy to $3.6 \AA$ resolution in an identical $P 2{ }_{1}$ unit cell (Table 3). X-ray diffraction data were indexed and scaled using iMOSFLM and treated for anisotropic diffraction using ellipsoidal truncation on the UCLA diffraction anisotropy server (services.mbi. ucla.edu/anisoscale). Phase information was determined using TOG1 (PDB ID:4FFB), TOG2 (PDB ID:4U3J), $\alpha \beta$-tubulin dimer, and DRP (PDB ID:4DRX) models using molecular replacement. Briefly, a truncated poly-alanine TOG domain including only its HEAT repeats was used in the molecular replacement rotation and translation search (Figure 2-figure supplement 1B-C). Eight $\alpha \beta$-tubulin and TOG domain solutions were identified based on the Log Likelihood Gain (LLG) values (Figure 2-figure supplement $1 \mathrm{~B}-\mathrm{C}$ ). After eight cycles of density modification, the electron-density map revealed the TOG1 domains exclusively due to the unique $\mathrm{C}$-terminal linker and vertical helix densities (Figure 2-figure supplement 1D-E). Density for eight DRP molecules was identified and built. DRP molecules interacted only with their cognate $\beta$-tubulin and did not form interfaces with $\alpha$ tubulin from neighboring molecules (Figure 2-figure supplement 1H). Two 2:4:4 sk-Alp14-monomer: $\alpha \beta$-tubulin:DRP wheel-like models were built and subjected to cycles of rigid-body refinement and model building using the $S$. kluyveri ortholog sequence. Each asymmetric unit contained two wheel-like assemblies (Figure 2-figure supplement 1E). TOG1-TOG2 linker residues (residues 265-299 in native sk-Alp14 and residues 260-277 in sk-Alp14-SL) were not observed and were presumed to be disordered (Figure 2-figure supplement 1F-H). Density maps from each of the wheel-like models were averaged using non-crystallographic symmetry and then refined using the PHENIX program (Adams et alo, 2010). Initially, models were refined using non-crystallographic symmetry (16 fold NCS) restraints and strictly constrained coordinates with group B-factor schemes. In the final stage refinement, the strategy was switched to individual positional and isotropic B-factor with automatic weight optimization. A $4.4 \AA$ sk-Alp14-monomer: $\alpha \beta$-tubulin:DRP structure and $3.6 \AA$ sk-Alp14-monomer-SL: $\alpha \beta$-tubulin:DRP structure are reported; refinement statistics appear in Table 3.

Rectangular crystals formed from sk-Alp14-monomer: $\alpha \beta$-tubulin: DRP $\Delta \mathrm{N}$ diffracted to $3.2 \AA$ resolution at the Argonne National Laboratory at the Advanced Photon Source microfocus APS 24-ID-C beamline. X-ray diffraction data were indexed in the $P 2_{1}$ space group with unique unit cell dimensions $a=115 \AA, b=194 \AA$, and $c=149 \AA$, with two complexes in each unit cell (Table 3). Phase information was determined using molecular replacement using the TOG1 and TOG2 domains and curved $\alpha \beta$-tubulin as search models (Figure 5 -figure supplement 1B). TOG1 and TOG2 domains were identified after cycles of density modification as described above. Four $\alpha \beta$-tubulins, four TOG domains, and two DRP $\triangle \mathrm{N}$ models were placed in the unit cell. The identity of TOG domains was determined using the conserved $\mathrm{C}$-terminal linker and jutting helix in the TOG1 domain sequence. A single DRP $\Delta N$ molecule was identified bound per two $\alpha \beta$-tubulin polymerized complex. Data from each extended assembly were combined using non-crystallographic symmetry (8-fold NCS) and were averaged and refined using the program PHENIX (Adams et al,, 2010) (Table 3). The individual positional coordinates and anisotropic B-factor were refined with automatic weight optimization in the final stage. A $3.2 \AA$ resolution refined density map is presented in Figure 5-figure supplement $1 C$. Examining data quality of sk-Alp14-monomer: $\alpha \beta$-tubulin:DRP or sk-Alp14-monomer: $\alpha \beta$ tubulin:DRP $\triangle N$ using PHENIX (Adams et alo, 2010) indicated that the diffraction data contained a small degree of pseudo-merohedral twinning. The twin fractions were adjusted during refinement of both models.

\section{Cysteine mutagenesis and crosslinking analyses of sk-Alp14: $\alpha \beta$-tubulin assemblies}

Based on the sk-Alp14-monomer: $\alpha \beta$-tubulin:DRP crystal structure, the $S$. kluyveri ortholog protein sk-Alp14, in its dimer form (residues 1-724), was used to generate crosslinking mutations. Interface 1 residues, which are in close proximity to each other, were mutated to cysteine: Ser180Cys (S180C) and Leu304Cys (L304C), which we termed S180C-L304C. Interface 2 residues, which are in close proximity to each other, were also mutated to cysteine: Ser41Cys (S41C) and Glu518Cys (E518C), which we termed S41C-E518C. The S. kluyveri ortholog dimer S180C-L304C mutant and S41CE518C mutant proteins were purified as described above (Figure 3E). These constructs were used either directly or to make complexes with $\alpha \beta$-tubulin in a 2:4 (subunit: $\alpha \beta$-tubulin) molar ratio, as 
described in Figure 1A. These S180C-L304C and S41C E518C mutants or their $\alpha \beta$-tubulin complexes were then treated using $5 \mathrm{mM} \mathrm{Cu}$-phenanthroline in $50 \mathrm{mM} \mathrm{HEPES}$ and $100 \mathrm{mM} \mathrm{KCl}, \mathrm{pH} 7.0$, for $5 \mathrm{~min}$, then treated with $5 \mathrm{mM}$ EDTA. These protein mixtures were subjected to SDS-PAGE under oxidizing conditions.

For LC/MS-MS mass spectrometry-based disulfide peptide mapping, S180C-L304C sk-Alp14 oxidized SDS-PAGE bands were subjected to in-gel proteolysis using either trypsin or chymotrypsin. Fragmented peptides were then purified and treated with $5 \mathrm{mM}$ iodioacetamide, which covalently adds $57 \mathrm{Da}$ in mass onto reduced cysteine-containing peptides, and does not affect cysteines locked in disulfides. The peptide mixture was then treated with $5 \mathrm{mM}$ dithiothriatol to reduce disulfides and then treated with 5-vinyl chloride, which covalently adds 105 Da mass units onto newly reduced cysteine-containing peptides. LCMS/MS mass spectrometry was performed and the resulting peptides were analyzed. Peptides covering $90 \%$ of sk-Alp14 were identified as were the majority of cysteines. Only two peptides were identified with cysteine residues that included $105 \mathrm{Da}$ mass units added as described in Figure 3-figure supplement $1 B$.

\section{Negative stain electron microscopy and image analysis of wt-Alp14 and TOG inactivated mutants in complex with $\alpha \beta$-tubulins}

SEC-purified $\alpha \beta$-tubulin complexes of wt-Alp14-dimer, INT1, INT2, and INT1 +2 at $100 \mathrm{mM} \mathrm{KCl} \mathrm{sup-}$ plemented with glutaraldehyde $0.05 \%$ and $4: 2$ molar ratio were placed on glow discharged grids, blotted after 30-60 s, and then stained with multiple washes of $0.1 \%$ uranyl formate at $\mathrm{pH}$ 7.0. All the images for negatively stained specimens were grids were collected on an electron microscope (JEM-2100F; JEOL) equipped with a field emission gun using low-dose mode at $200 \mathrm{KeV}$ paired with a DE-20 direct electron detector device (DDD) operating in integration mode. The images were then processed using neural networks picking using Cr-YOLO (Wagner et al, https://doi.org/10.1101/ 356584). The particle coordinates were imported into relion 2.2. Images were CTF corrected using CTFFIND4 (Grant et al,). Particles were manually screened and subjected to rounds of 2D-classification either in Relion 2.2 or using Cryosparc (Punjani et al., 2017; Kimanius et al., 2016). For each data set, 2D-Class averages were grouped based on their conformation then compared to $30 \AA$ resolution filtered models of 4:2 $\alpha \beta$-tubulin: TOG2-TOG2 in square conformation (Figure 2 ) and single TOG1-TOG2 subunit from the square conformation in the bent conformation bound to two nonpolymerized $\alpha \beta$-tubulins (Figure 6), TOG1-TOG2 in the polymerized conformation (Figure 5) and a single TOG domain bound $\alpha \beta$-tubulin (PDB-ID:4FFD). The projection matching was performed using EMAN2 command e2classvsproj.py at 1-5 angular degree increments (Tang et al., 2007).

\section{Animating the MT polymerase 'polarized unfurling' mechanism}

The animation was created using BLENDER 3D-animation software (http://blender.org) as follows. Briefly, surface and ribbon models of PDB coordinates representing the structures were exported from UCSF-Chimera and imported into BLENDER, and then smoothed and optimized to generate animated models. Additional protein SK-rich regions and coiled-coil domains, whose structures are unknown, were thus modeled using sequence length and other information as guidance. The microtubule lattice was modeled based on the tubulin structure (PDB ID 3J6F). The dissociation of TOG1 and TOG2 domains from $\alpha \beta$-tubulins were simulated in the animation, based on biochemical studies described in Figure 1 and its figure supplements.

\section{Acknowledgements}

We thank Dr. Julian Eskin (Brandeis University) for animating the microtubule polymerase mechanism. We thank Advanced Photon Source (APS) and Drs. K Rajashankar, J Schuermann, N Sukumar, and D Neau of the Northeastern Collaborative Access Team (NE-CAT) for use of the 24-ID-C and ID-E beam lines to collect all X-ray diffraction data for our crystallographic studies. We thank Dr. Christopher Fraser (UC Davis) for using his Nano-ITC. We thank Dr. Rick Mckenney (UC Davis), and Dr. Kevin Corbett (UC San Diego) for advice and critical reading of this manuscript. JAB and FC are supported by National Institutes of Health GM110283 and GM115185, respectively. This work is based upon research conducted at the NE-CAT beamlines, which are funded by the National Institute of General Medical Sciences from the National Institutes of Health (P41 GM103403). The Pilatus $6 \mathrm{M}$ detector on 24-ID-C beam line is funded by a NIH-ORIP HEI grant (S10 RR029205). The Eiger 
$16 \mathrm{M}$ detector on 24-ID-E beam line is funded by a NIH-ORIP HEI grant (S100D021527). This research used resources of the Advanced Photon Source, a US Department of Energy (DOE) Office of Science User Facility operated for the DOE Office of Science by Argonne National Laboratory under Contract No. DE-AC02-06CH11357. sk-Alp14-monomer: $\alpha \beta$-tubulin:DRP, sk-Alp14-monomerSL: $\alpha \beta$-tubulin:DRP, and sk-Alp14-monomer: $\alpha \beta$-tubulin:DRP $\Delta \mathrm{N}$ are available at the protein data bank (PDB) under PDB-ID 6MZF, PDB-ID 6MZE and PDB-ID 6MZG, respectively.

\section{Additional information}

Funding

Funder

Grant reference number Author

\begin{tabular}{lll}
\hline National Science Foundation & MCB1615991 & Jawdat Al-Bassam \\
\hline National Institutes of Health & GM115185 & Jawdat Al-Bassam \\
\hline National Institutes of Health & GM110283 & Jawdat Al-Bassam
\end{tabular}

The funders had no role in study design, data collection and interpretation, or the decision to submit the work for publication.

Author contributions

Stanley Nithianantham, Conceptualization, Data curation, Software, Formal analysis, Supervision, Validation, Investigation, Visualization, Methodology, Writing-original draft, Writing-review and editing; Brian D Cook, Conceptualization, Resources, Data curation, Software, Formal analysis, Supervision, Funding acquisition, Validation, Investigation, Visualization, Methodology, Writingreview and editing; Madeleine Beans, Fei Guo, Data curation; Fred Chang, Conceptualization, Supervision, Funding acquisition, Validation, Methodology, Writing-original draft, Project administration, Writing-review and editing; Jawdat Al-Bassam, Conceptualization, Resources, Data curation, Software, Formal analysis, Supervision, Funding acquisition, Validation, Investigation, Visualization, Methodology, Writing-original draft, Project administration, Writing-review and editing

Author ORCIDs

Stanley Nithianantham (D) http://orcid.org/0000-0001-6238-647X

Jawdat Al-Bassam (D) http://orcid.org/0000-0001-6625-2102

Decision letter and Author response

Decision letter https://doi.org/10.7554/eLife.38922.035

Author response https://doi.org/10.7554/eLife.38922.036

\section{Additional files}

Supplementary files

- Transparent reporting form

DOl: https://doi.org/10.7554/eLife.38922.027

Data availability

All data generated or analyzed during this study are included in the manuscript and supporting files.

The following datasets were generated:

\begin{tabular}{|c|c|c|c|c|}
\hline Author(s) & Year & Dataset title & Dataset URL & $\begin{array}{l}\text { Database and } \\
\text { Identifier }\end{array}$ \\
\hline $\begin{array}{l}\text { Stanley Nithianan- } \\
\text { tham, Brian D Cook, } \\
\text { Madielene Beans, } \\
\text { Fei Guo, Fred } \\
\text { Chang, Jawdat Al- }\end{array}$ & 2018 & $\begin{array}{l}\text { 1:2:2 sk-Alp14-momomer: } \alpha \beta \text { - } \\
\text { Tubulin:DRP }\end{array}$ & $\begin{array}{l}\text { https://www.rcsb.org/ } \\
\text { structure/6MZF }\end{array}$ & $\begin{array}{l}\text { RCSB Protein Data } \\
\text { Bank, 6MZF }\end{array}$ \\
\hline
\end{tabular}


Bassam

\begin{tabular}{|c|c|c|c|c|}
\hline $\begin{array}{l}\text { Stanley Nithianan- } \\
\text { tham, Brian D Cook, } \\
\text { Madielene Beans, } \\
\text { Fei Guo, Fred } \\
\text { Chang, Jawdat Al- } \\
\text { Bassam }\end{array}$ & 2018 & $\begin{array}{l}\text { 1:2:2 sk-Alp14-monomer-SL: } \alpha \beta \text { - } \\
\text { Tubulin:DRP }\end{array}$ & $\begin{array}{l}\text { https://www.rcsb.org/ } \\
\text { structure/6MZE }\end{array}$ & $\begin{array}{l}\text { RCSB Protein Data } \\
\text { Bank, 6MZE }\end{array}$ \\
\hline $\begin{array}{l}\text { Stanley Nithianan- } \\
\text { tham, Brian D Cook, }\end{array}$ & 2018 & $\begin{array}{l}\text { 1:2:1 sk-Alp14-monomer: } \alpha \beta- \\
\text { Tubulin:DRP } \Delta N\end{array}$ & $\begin{array}{l}\text { https://www.rcsb.org/ } \\
\text { structure/6MZG }\end{array}$ & $\begin{array}{l}\text { RCSB Protein Data } \\
\text { Bank, 6MZG }\end{array}$ \\
\hline
\end{tabular}
Tubulin:DRP $\Delta N$ structure/6MZG

Bank, 6MZG

Fei Guo, Fred

Chang, Jawdat Al-

Bassam

\section{References}

Adams PD, Afonine PV, Bunkóczi G, Chen VB, Davis IW, Echols N, Headd JJ, Hung LW, Kapral GJ, GrosseKunstleve RW, McCoy AJ, Moriarty NW, Oeffner R, Read RJ, Richardson DC, Richardson JS, Terwilliger TC, Zwart PH. 2010. PHENIX: a comprehensive Python-based system for macromolecular structure solution. Acta Crystallographica Section D Biological Crystallography 66:213-221. DOI: https://doi.org/10.1107/ S0907444909052925, PMID: 20124702

Akhmanova A, Steinmetz MO. 2008. Tracking the ends: a dynamic protein network controls the fate of microtubule tips. Nature Reviews Molecular Cell Biology 9:309-322. DOI: https://doi.org/10.1038/nrm2369, PMID: 18322465

Akhmanova A, Steinmetz MO. 2011. Microtubule end binding: EBs sense the guanine nucleotide state. Current Biology 21:R283-R285. DOI: https://doi.org/10.1016/j.cub.2011.03.023, PMID: 21514511

Akhmanova A, Steinmetz MO. 2015. Control of microtubule organization and dynamics: two ends in the limelight. Nature Reviews Molecular Cell Biology 16:711-726. DOl: https://doi.org/10.1038/nrm4084, PMID: 26562752

Al-Bassam J, van Breugel M, Harrison SC, Hyman A. 2006. Stu2p binds tubulin and undergoes an open-to-closed conformational change. The Journal of Cell Biology 172:1009-1022. DOI: https://doi.org/10.1083/jcb. 200511010, PMID: 16567500

Al-Bassam J, Larsen NA, Hyman AA, Harrison SC. 2007. Crystal structure of a TOG domain: conserved features of XMAP215/Dis1-family TOG domains and implications for tubulin binding. Structure 15:355-362. DOI: https://doi.org/10.1016/j.str.2007.01.012, PMID: 17355870

Al-Bassam J, Kim H, Brouhard G, van Oijen A, Harrison SC, Chang F. 2010. CLASP promotes microtubule rescue by recruiting tubulin dimers to the microtubule. Developmental Cell 19:245-258. DOI: https://doi.org/10.1016/ j.devcel.2010.07.016, PMID: 20708587

Al-Bassam J, Chang F. 2011. Regulation of microtubule dynamics by TOG-domain proteins XMAP215/Dis1 and CLASP. Trends in Cell Biology 21:604-614. DOI: https://doi.org/10.1016/j.tcb.2011.06.007, PMID: 21782439

Al-Bassam J, Kim H, Flor-Parra I, Lal N, Velji H, Chang F. 2012. Fission yeast Alp14 is a dose-dependent plus end-tracking microtubule polymerase. Molecular Biology of the Cell 23:2878-2890. DOI: https://doi.org/10. 1091/mbc.e12-03-0205, PMID: 22696680

Alushin GM, Lander GC, Kellogg EH, Zhang R, Baker D, Nogales E. 2014. High-resolution microtubule structures reveal the structural transitions in $\alpha \beta$-tubulin upon GTP hydrolysis. Cell 157:1117-1129. DOI: https://doi.org/ 10.1016/j.cell.2014.03.053, PMID: 24855948

Ayaz P, Ye X, Huddleston P, Brautigam CA, Rice LM. 2012. A TOG: $\alpha \beta$-tubulin complex structure reveals conformation-based mechanisms for a microtubule polymerase. Science 337:857-860. DOI: https://doi.org/10. 1126/science.1221698, PMID: 22904013

Ayaz P, Munyoki S, Geyer EA, Piedra FA, Vu ES, Bromberg R, Otwinowski Z, Grishin NV, Brautigam CA, Rice LM. 2014. A tethered delivery mechanism explains the catalytic action of a microtubule polymerase. eLife 3:e03069. DOI: https://doi.org/10.7554/eLife.03069, PMID: 25097237

Battye TG, Kontogiannis L, Johnson O, Powell HR, Leslie AG. 2011. iMOSFLM: a new graphical interface for diffraction-image processing with MOSFLM. Acta Crystallographica. Section D, Biological Crystallography 67: 271-281. DOI: https://doi.org/10.1107/S0907444910048675, PMID: 21460445

Brouhard GJ, Stear JH, Noetzel TL, Al-Bassam J, Kinoshita K, Harrison SC, Howard J, Hyman AA. 2008. XMAP215 is a processive microtubule polymerase. Cell 132:79-88. DOI: https://doi.org/10.1016/j.cell.2007.11. 043, PMID: 18191222

Brouhard GJ, Rice LM. 2014. The contribution of $\alpha \beta$-tubulin curvature to microtubule dynamics. The Journal of Cell Biology 207:323-334. DOI: https://doi.org/10.1083/jcb.201407095, PMID: 25385183

Castoldi M, Popov AV. 2003. Purification of brain tubulin through two cycles of polymerization-depolymerization in a high-molarity buffer. Protein Expression and Purification 32:83-88. DOI: https://doi.org/10.1016/S10465928(03)00218-3, PMID: 14680943

Chen VB, Arendall WB, Headd JJ, Keedy DA, Immormino RM, Kapral GJ, Murray LW, Richardson JS, Richardson DC. 2012. MolProbity: all-atom structure validation for macromolecular crystallography. International Tables for Crystallography:694-701. 
Cowtan KD, Main P. 1996. Phase combination and cross validation in iterated density-modification calculations. Acta Crystallographica Section D Biological Crystallography 52:43-48. DOI: https://doi.org/10.1107/ S090744499500761X, PMID: 15299724

Cullen CF, Deák P, Glover DM, Ohkura H. 1999. mini spindles: A gene encoding a conserved microtubuleassociated protein required for the integrity of the mitotic spindle in Drosophila. The Journal of Cell Biology 146:1005-1018. DOI: https://doi.org/10.1083/jcb.146.5.1005, PMID: 10477755

Das A, Dickinson DJ, Wood CC, Goldstein B, Slep KC. 2015. Crescerin uses a TOG domain array to regulate microtubules in the primary cilium. Molecular Biology of the Cell 26:4248-4264. DOI: https://doi.org/10.1091/ mbc.e15-08-0603, PMID: 26378256

Emsley P, Lohkamp B, Scott WG, Cowtan K. 2010. Features and development of Coot. Acta Crystallographica. Section D, Biological Crystallography 66:486-501. DOI: https://doi.org/10.1107/S0907444910007493, PMID: 20383002

Fox JC, Howard AE, Currie JD, Rogers SL, Slep KC. 2014. The XMAP215 family drives microtubule polymerization using a structurally diverse TOG array. Molecular Biology of the Cell 25:2375-2392. DOI: https://doi.org/10.1091/mbc.e13-08-0501, PMID: 24966168

Geyer EA, Miller MP, Brautigam CA, Biggins S, Rice LM. 2018. Design principles of a microtubule polymerase. eLife 7:e34574. DOI: https://doi.org/10.7554/eLife.34574, PMID: 29897335

Howard AE, Fox JC, Slep KC. 2015. Drosophila melanogaster mini spindles TOG3 utilizes unique structural elements to promote domain stability and maintain a TOG1- and TOG2-like tubulin-binding surface. Journal of Biological Chemistry 290:10149-10162. DOI: https://doi.org/10.1074/jbc.M114.633826, PMID: 25720490

Kelley LA, Mezulis S, Yates CM, Wass MN, Sternberg MJ. 2015. The Phyre2 web portal for protein modeling, prediction and analysis. Nature Protocols 10:845-858. DOI: https://doi.org/10.1038/nprot.2015.053, PMID: 25 950237

Kimanius D, Forsberg BO, Scheres SH, Lindahl E. 2016. Accelerated cryo-EM structure determination with parallelisation using GPUs in RELION-2. eLife 5:e18722. DOI: https://doi.org/10.7554/eLife.18722, PMID: 27 845625

Lechner B, Rashbrooke MC, Collings DA, Eng RC, Kawamura E, Whittington AT, Wasteneys GO. 2012. The $\mathrm{N}$-terminal TOG domain of Arabidopsis MOR1 modulates affinity for microtubule polymers. Journal of Cell Science 125:4812-4821. DOI: https://doi.org/10.1242/jcs.107045, PMID: 22825869

Maurer SP, Cade NI, Bohner G, Gustafsson N, Boutant E, Surrey T. 2014. EB1 accelerates two conformational transitions important for microtubule maturation and dynamics. Current Biology 24:372-384. DOI: https://doi. org/10.1016/j.cub.2013.12.042, PMID: 24508171

McCoy AJ. 2007. Solving structures of protein complexes by molecular replacement with Phaser. Acta Crystallographica Section D Biological Crystallography 63:32-41. DOI: https://doi.org/10.1107/ S0907444906045975, PMID: 17164524

Miller MP, Asbury CL, Biggins S. 2016. A TOG Protein Confers Tension Sensitivity to Kinetochore-Microtubule Attachments. Cell 165:1428-1439. DOI: https://doi.org/10.1016/j.cell.2016.04.030, PMID: 27156448

Nawrotek A, Knossow M, Gigant B. 2011. The determinants that govern microtubule assembly from the atomic structure of GTP-tubulin. Journal of Molecular Biology 412:35-42. DOI: https://doi.org/10.1016/j.jmb.2011.07 029, PMID: 21787788

Pecqueur L, Duellberg C, Dreier B, Jiang Q, Wang C, Plückthun A, Surrey T, Gigant B, Knossow M. 2012. A designed ankyrin repeat protein selected to bind to tubulin caps the microtubule plus end. PNAS 109:1201112016. DOI: https://doi.org/10.1073/pnas.1204129109, PMID: 22778434

Pettersen EF, Goddard TD, Huang CC, Couch GS, Greenblatt DM, Meng EC, Ferrin TE. 2004. UCSF Chimera-a visualization system for exploratory research and analysis. Journal of Computational Chemistry 25:1605-1612. DOI: https://doi.org/10.1002/jcc.20084, PMID: 15264254

Punjani A, Rubinstein JL, Fleet DJ, Brubaker MA. 2017. cryoSPARC: algorithms for rapid unsupervised cryo-EM structure determination. Nature Methods 14:290-296. DOI: https://doi.org/10.1038/nmeth.4169, PMID: 2 8165473

Reber SB, Baumgart J, Widlund PO, Pozniakovsky A, Howard J, Hyman AA, Jülicher F. 2013. XMAP215 activity sets spindle length by controlling the total mass of spindle microtubules. Nature Cell Biology 15:1116-1122. DOI: https://doi.org/10.1038/ncb2834, PMID: 23974040

Slep KC, Vale RD. 2007. Structural basis of microtubule plus end tracking by XMAP215, CLIP-170, and EB1. Molecular Cell 27:976-991. DOI: https://doi.org/10.1016/j.molcel.2007.07.023, PMID: 17889670

Strong M, Sawaya MR, Wang S, Phillips M, Cascio D, Eisenberg D. 2006. Toward the structural genomics of complexes: Crystal structure of a PE/PPE protein complex from Mycobacterium tuberculosis. PNAS 103:80608065. DOI: https://doi.org/10.1073/pnas.0602606103

Tanaka K, Mukae N, Dewar H, van Breugel M, James EK, Prescott AR, Antony C, Tanaka TU. 2005. Molecular mechanisms of kinetochore capture by spindle microtubules. Nature 434:987-994. DOI: https://doi.org/10 1038/nature03483, PMID: 15846338

Tang G, Peng L, Baldwin PR, Mann DS, Jiang W, Rees I, Ludtke SJ. 2007. EMAN2: an extensible image processing suite for electron microscopy. Journal of Structural Biology 157:38-46. DOI: https://doi.org/10 1016/j.jsb.2006.05.009, PMID: 16859925

Terwilliger TC. 2000. Maximum-likelihood density modification. Acta Crystallographica Section D Biological Crystallography 56:965-972. DOI: https://doi.org/10.1107/S0907444900005072, PMID: 10944333

Wagner T, Merino F, Stabrin M, Moriya T, Gatsogiannis C, Raunser S. 2018. SPHIRE-crYOLO: A fast and wellcentering automated particle picker for cryo-EM. Biorxiv. DOI: https://doi.org/10.1101/356584 
Wang PJ, Huffaker TC. 1997. Stu2p: A microtubule-binding protein that is an essential component of the yeast spindle pole body. The Journal of Cell Biology 139:1271-1280. DOI: https://doi.org/10.1083/jcb.139.5.1271, PMID: 9382872

Widlund PO, Stear JH, Pozniakovsky A, Zanic M, Reber S, Brouhard GJ, Hyman AA, Howard J. 2011. XMAP215 polymerase activity is built by combining multiple tubulin-binding TOG domains and a basic lattice-binding region. PNAS 108:2741-2746. DOI: https://doi.org/10.1073/pnas.1016498108, PMID: 21282620 